\title{
SOME NEW DE MORGAN PICTURE OPERATOR TRIPLES IN PICTURE FUZZY LOGIC
}

\author{
BUI CONG CUONG ${ }^{1}$, ROAN THI NGAN ${ }^{2}$, LE BA LONG ${ }^{3}$ \\ ${ }^{1}$ Institute of Mathematics; ${ }^{1}$ bccuong@gmail.com \\ ${ }^{2}$ Basic Science Faculty, Ha Noi University of Natural Resources and Environment \\ ${ }^{3}$ Post and Telecommunication Institute of Technology
}

\begin{abstract}
A new concept of picture fuzzy sets (PFS) were introduced in 2013, which are direct extensions of the fuzzy sets and the intuitionistic fuzzy sets. Then some operations on PFS with some properties are considered in $[7,5]$. Some basic operators of fuzzy logic as negation, t-norms, t-conorms for picture fuzzy sets firstly are defined and studied in [6,9]. This paper is devoted to some classes of representable picture fuzzy t-norms and representable picture fuzzy t-conorms on PFS and a basic algebra structure of Picture Fuzzy Logic De Morgan triples of picture operators.
\end{abstract}

Keywords. Picture fuzzy sets, Picture fuzzy t-norms, Picture fuzzy t-conorm, De Morgan picture operator triple.

\section{INTRODUCTION}

Recently, Bui Cong Cuong and Kreinovich (2013) first defined "picture fuzzy sets" [7, 5], which are a generalization of the Zadeh's fuzzy sets [27, 28] and the Antanassov's intuitionistic fuzzy sets $[2,1]$. This concept is particularly effective in approaching the practical problems in relation to the synthesis of ideas, such as decisions making problems, voting analysis, fuzzy clustering, financial forecasting. The basic definitions and basic operators in the picture fuzzy sets theory were given in $[3,4,7,5]$. The new basic logic connectives on the PFS firstly were presented in $[6,9]$. These new concepts are supporting to new computing procedures in computational intelligence problems and in other applications (see $[14,15,16,17,18,19,20,21,23,24,25,26])$.

In this paper we study some algebraic properties of the picture fuzzy t-norms and the picture fuzzy t-conorms on PFS, which are basic operators of the Picture Fuzzy Logics. Some classes of the representable picture fuzzy t-norms and the representable picture fuzzy t-conorms were first given in $[1,8]$ will be presented. Then a basic algebra structure on PFS - De Morgan picture operator triples will be considered and some new De Morgan picture operator triples will be presented.

We first recall some basic notions of the picture fuzzy sets.

Definition 1.1. [7] A picture fuzzy set $A$ on a universe $X$ is an object of the form

$$
A=\left\{\left(x, \mu_{A}(x), \eta_{A}(x), \nu_{A}(x)\right) \mid x \in X\right\},
$$

(c) 2017 Vietnam Academy of Science \& Technology 
where $\mu_{A}(x), \eta_{A}(x), \nu_{A}(x)$ are respectively called the degree of positive membership, the degree of neutral membership, the degree of negative membership of $\mathrm{x}$ in $\mathrm{A}$, and the following conditions are satisfied:

$$
0 \leq \mu_{A}(x), \eta_{A}(x), \nu_{A}(x) \leq 1 \text { and } \mu_{A}(x)+\eta_{A}(x)+\nu_{A}(x) \leq 1, \forall x \in X .
$$

Then, $\forall x \in X: \quad 1-\left(\mu_{A}(x)+\eta_{A}(x)+\nu_{A}(x)\right)$ is called the degree of refusal membership of $x$ in $A$.

Consider the set $D^{*}=\left\{x=\left(x_{1}, x_{2}, x_{3}\right) \mid x \in[0,1]^{3}, x_{1}+x_{2}+x_{3} \leq 1\right\}$. From now on, we will assume that if $x \in D^{*}$, then $x_{1}, x_{2}$ and $x_{3}$ denote, respectively, the first, the second and the third component of $x$, i.e. , $x=\left(x_{1}, x_{2}, x_{3}\right)$.

We have a lattice $\left(D^{*}, \leq_{1}\right)$, where $\leq_{1}$ defined for $\forall x, y \in D^{*}$

$$
\begin{gathered}
\left(x \leq_{1} y\right) \Leftrightarrow\left(x_{1}<y_{1}, x_{3} \geq y_{3}\right) \vee\left(x_{1}=y_{1}, x_{3}>y_{3}\right) \vee\left(\left\{x_{1}=y_{1}, x_{3}=y_{3}, x_{2} \leq y_{2}\right\}\right), \\
(x=y) \Leftrightarrow\left(x_{1}=y_{1}, x_{2}=y_{2}, x_{3}=y_{3}\right), \quad \forall x, y \in D^{*}
\end{gathered}
$$

We define the first, second and third projection mapping $p r_{1}, p r_{2}$ and $p r_{3}$ on $D^{*}$ as $p r_{1}(x)=x_{1}$ and $p r_{2}(x)=x_{2}$ and $p r_{3}(x)=x_{3}$, on all $x \in D^{*}$.

Note that, if for $x, y \in D^{*}$ that neither $x \leq_{1} y$ nor $y \leq_{1} x$, then $x$ and $y$ are incomparable w.r.t $\leq_{1}$, and denoted as $x \|_{\leq_{1}} y$.

From now on, we denote $u \wedge v=\min (u, v), u \vee v=\max (u, v)$ for all $u, v \in R^{1}$.

For each $x, y \in D^{*}$, we define

$$
\begin{gathered}
\inf (x, y)=\left\{\begin{array}{l}
\min (x, y), \quad \text { if } \quad x \leq_{1} y \text { or } y \leq_{1} x \\
\left(x_{1} \wedge y_{1}, 1-x_{1} \wedge y_{1}-x_{3} \vee y_{3}, x_{3} \vee y_{3}\right), \quad \text { else }
\end{array}\right. \\
\sup (x, y)=\left\{\begin{array}{l}
\max (x, y), \quad \text { if } \quad x \leq_{1} y \text { or } y \leq_{1} x \\
\left(x_{1} \vee y_{1}, 0, x_{3} \wedge y_{3}\right), \quad \text { else }
\end{array}\right.
\end{gathered}
$$

Proposition 1.2. With these operators $\left(D^{*}, \leq_{1}\right)$ is a complete lattice.

Proof. See $[6,9]$.

Using this lattice, we easily see that every picture fuzzy set

$$
A=\left\{\left(x, \mu_{A}(x), \eta_{A}(x), \nu_{A}(x)\right) \mid x \in X\right\}
$$

corresponds an $D^{*}$ - fuzzy set [12] mapping, i.e., we have a mapping

$$
A: X \rightarrow D^{*}: x \rightarrow\left\{\left(x, \mu_{A}(x), \eta_{A}(x), \nu_{A}(x)\right) \mid x \in X\right\} .
$$

Interpreting picture fuzzy sets as $D^{*}$-fuzzy sets gives way to greater flexibility in calculating with membership degrees, since the triple of numbers formed by the three degrees is an element of $D^{*}$, and often allows to obtain more compact formulas. 


\section{PICTURE FUZZY NEGATION OPERATOR}

Now we consider some basic fuzzy operators of the Picture Fuzzy Logics.

Picture fuzzy negations are an extension of the fuzzy negations [22] and the intuitionistic fuzzy negations [2]. They are defined as follows.

Definition 2.1. A mapping $N: D^{*} \rightarrow D^{*}$ satisfying conditions $N\left(0_{D^{*}}\right)=1_{D^{*}}$ and $N\left(1_{D^{*}}\right)=$ $0_{D^{*}}$ and $N$ is nonincreasing is called a picture fuzzy negation operator.

If $N(N(x))=x$ for all $x \in D^{*}$, then $N$ is called an involutive negation operator.

Definition 2.2. Let $f_{1}, f_{2}: D^{*} \rightarrow D^{*}$ be mappings on $D^{*}$. We say that the mapping $f_{2}$ is greater than $f_{1}$ if $f_{1}(x) \leq_{1} f_{2}(x), \forall x \in D^{*}$, and we denote that as $f_{1} \leq f_{2}$. We write $f_{1}<f_{2}$, if $f_{1} \leq f_{2}$, and $f_{1} \neq f_{2}$.

Let $x=\left(x_{1}, x_{2}, x_{3}\right) \in D^{*}$. We first give 2 drastic picture negation operators

$n_{d}(x)=\left\{\begin{array}{l}0_{D^{*}} \text { if } x \neq 0_{D^{*}} \\ 1_{D^{*}} \text { if } x=0_{D^{*}}\end{array}\right.$ and $n_{d 2}(x)=\left\{\begin{array}{l}1_{D^{*}} \text { if } x \neq 1_{D^{*}} \\ 0_{D^{*}} \text { if } x=1_{D^{*}} .\end{array}\right.$

Proposition 2.3. $n_{d}$ and $n_{d 2}$ are picture negation operators and for each picture negation operator $n(x), n_{d}(x) \leq_{1} n(x) \leq_{1} n_{d 2}(x), \forall x \in D^{*}$.

Definition 2.4. The mapping $n_{0}: D^{*} \rightarrow D^{*}$ defined by $n_{0}(x)=\left(x_{3}, 0, x_{1}\right)$, for each $x \in D^{*}$.

Proposition 2.5. $n_{0}$ is a picture fuzzy negation operator. It is called the simple picture negation.

Proof. Indeed, $1_{D^{*}}=(1,0,0) \in D^{*}$ then $n_{0}\left(1_{D^{*}}\right)=n_{0}(1,0,0)=(0,0,1)=0_{D^{*}}$. Analogously, $n_{0}\left(0_{D^{*}}\right)=n_{0}(0,0,1)=(1,0,0)=1_{D^{*}} \in D^{*}$.

Let $x, y \in D^{*}$ and $x \leq_{1} y$. Consider 3 subsets

$$
\begin{gathered}
B_{1}=\left\{\left(x_{1}<y_{1}\right) \wedge\left(x_{3} \geq y_{3}\right)\right\}, \\
B_{2}=\left\{\left(x_{1}=y_{1}\right) \wedge\left(x_{3}>y_{3}\right)\right\}, \\
B_{3}=\left\{\left(x_{1}=y_{1}\right) \wedge\left(x_{3}=y_{3}\right) \wedge\left(x_{2} \leq y_{2}\right)\right\} .
\end{gathered}
$$

We have to consider 4 following cases

Case 1a. $x_{1}<y_{1}$ and $x_{3}=y_{3}$ then $\left(n_{0}(y), n_{0}(x)\right) \in B_{2} \Rightarrow n_{0}(y) \leq{ }_{1} n_{0}(x)$,

Case 1b. $x_{1}<y_{1}$ and $x_{3}>y_{3}$ then $\left(n_{0}(y), n_{0}(x)\right) \in B_{1} \Rightarrow n_{0}(y) \leq{ }_{1} n_{0}(x)$,

Case 2. $x_{1}=y_{1}$ and $x_{3}>y_{3}$ then $\left(n_{0}(y), n_{0}(x)\right) \in B_{1} \Rightarrow n_{0}(y) \leq_{1} n_{0}(x)$,

Case 3. $x_{1}=y_{1}$ and $x_{3}=y_{3}$ then $\left(n_{0}(y), n_{0}(x)\right) \in B_{3} \Rightarrow n_{0}(y) \leq_{1} n_{0}(x)$.

It shows that the mapping $n_{0}(x)=\left(x_{3}, 0, x_{1}\right)$ is non-increasing and the operator $n_{0}(x)$ is a picture negation operator.

Definition 2.6. Let $x=\left(x_{1}, x_{2}, x_{3}\right) \in D^{*}$. Denote $x_{4}=1-\left(x_{1}+x_{2}+x_{3}\right)$. The mapping $N_{S}$ is by $N_{S}(x)=\left(x_{3}, x_{4}, x_{1}\right)$, for each $x \in D^{*}$.

Proposition 2.7. $N_{S}$ is an involutive picture negation operator and is called the picture standard negation operator.

Proof. It is analogous to the proof of the Proposition 2.5

Some other picture fuzzy negations were given in [9]. 


\section{PICTURE FUZZY T-NORMS AND PICTURE FUZZY T-CONORMS}

Fuzzy t-norms on $[0,1]$ and fuzzy t-conorms on $[0,1]$ were defined and considered in $[22,13]$.

In 2004 , G.Deschrijver et al. [10] introduced the notion of intuitionistic fuzzy t-norms and t-conorms and investigated under which conditions a similar representation theorem could be obtained. For further usage, we define $L^{*}=\left\{x \in D^{*} \mid x_{2}=0\right\}$.

We can consider the set $L^{*}$ defined by $L^{*}=\left\{u=\left(u_{1}, u_{3}\right) \mid u \in[0,1]^{2}, u_{1}+u_{3} \leq 1\right\}$.

Consider the order relation $u \leq v$ on $L^{*}$, defined by $u \leq v \Leftrightarrow\left(\left(u_{1} \leq v_{1}\right) \wedge\left(u_{3} \geq v_{3}\right)\right)$, for all $u, v \in L^{*}$.

We define the first, and the second projection mapping $p r_{1}$ and $p r_{3}$ on $L^{*}$, as $p r_{1}(u)=u_{1}$ and $p_{3}(u)=u_{3}$, on all $u \in L^{*}$. The units of $L^{*}$ are $1_{L^{*}}=(1,0)$ and $0_{L^{*}}=(0,1)$.

Definition 3.1. [10] An intuitionistic fuzzy t-norm is a commutative, associative, increasing $\left(L^{*}\right)^{2} \rightarrow L^{*}$ mapping $T$ satisfying $T\left(1_{L^{*}}, u\right)=u$, for all $u \in L^{*}$.

Definition 3.2. [10] An intuitionistic fuzzy t-conorm is a commutative, associative, increasing

$\left(L^{*}\right)^{2} \rightarrow L^{*}$ mapping $S$ satisfying $S\left(v, 0_{L^{*}}\right)=v$, for all $v \in L^{*}$.

Definition 3.3. [10] An intuitionistic fuzzy t-norm $T$ is called t-representable iff there exist a fuzzy t-norm $t_{1}$ on $[0,1]$ and a fuzzy t-conorm $s_{3}$ on $[0,1]$ satisfying for all $u, v \in L^{*}$,

$$
T(u, v)=\left(t_{1}\left(u_{1}, v_{1}\right), s_{3}\left(u_{3}, v_{3}\right)\right) .
$$

Definition 3.4. [10] An intuitionistic fuzzy t-conorm $S$ is called t-representable iff there exist a fuzzy t-norm $t_{1}$ on $[0,1]$ and a fuzzy t-conorm $s_{3}$ on $[0,1]$ satisfying for all $u, v \in L^{*}$,

$$
S(u, v)=\left(s_{3}\left(u_{1}, v_{1}\right), t_{1}\left(u_{3}, v_{3}\right)\right) .
$$

Now we define picture fuzzy t-norms and picture fuzzy t-conorms, which are classes of conjunction operators and classes of disjunction operators - main basic operators of the picture fuzzy logics. Picture fuzzy t-norms are direct extensions of the fuzzy t-norms in $[28,22,13]$ and of the intuitionistic fuzzy t-norms in [2], and they are important operators in $[11]$.

Let $x=\left(x_{1}, x_{2}, x_{3}\right) \in D^{*}$. Denote $I(x)=\left\{y \in D^{*}: y=\left(x_{1}, y_{2}, x_{3}\right), 0 \leq_{1} y_{2} \leq_{1} x_{2}\right\}$.

Definition 3.5. A mapping $T: D^{*} \times D^{*} \rightarrow D^{*}$ is a picture fuzzy t-norm if the mapping $T$ satisfies the following conditions

$T(x, y)=T(y, x), \forall x, y \in D^{*}$ (commutative),

$T(x, T(y, z))=T(T(x, y), z), \forall x, y, z \in D^{*}$ (associativity),

$T(x, y) \leq_{1} T(x, z), \forall x, y, z \in D^{*}, y \leq_{1} z$ (monotonicity),

$T\left(1_{D^{*}}, x\right) \in I(x), \forall x \in D^{*}$ (boundary condition).

Fisrt we present some picture fuzzy t-norms on picture fuzzy sets.

Definition 3.6. A picture fuzzy t-norm $T$ is called representable iff there exist two fuzzy t-norms $t_{1}, t_{2}$ on $[0,1]$ and a fuzzy t-conorm $s_{3}$ on $[0,1]$ satisfying

$$
T(x, y)=\left(t_{1}\left(x_{1}, y_{1}\right), t_{2}\left(x_{2}, y_{2}\right), s_{3}\left(x_{3}, y_{3}\right)\right), \forall x, y \in D^{*}
$$


We give some representable picture fuzzy t-norms, for all $x, y \in D^{*}$

1. $T_{\min }(x, y)=\left(\min \left(x_{1}, y_{1}\right), \min \left(x_{2}, y_{2}\right), \max \left(x_{3}, y_{3}\right)\right)$.

2. $T_{02}(x, y)=\left(\min \left(x_{1}, y_{1}\right), x_{2} y_{2}, \max \left(x_{3}, y_{3}\right)\right)$.

3. $T_{03}(x, y)=\left(x_{1} y_{1}, x_{2} y_{2}, \max \left(x_{3}, y_{3}\right)\right)$.

4. $T_{04}(x, y)=\left(x_{1} y_{1}, x_{2} y_{2}, x_{3}+y_{3}-x_{3} y_{3}\right)$.

5. $T_{05}(x, y)=$

$$
\left(\left\{\begin{array}{l}
x_{1} \wedge y_{1} \text { if } x_{1} \vee y_{1}=1 \\
0 i f x_{1} \vee y_{1}<1
\end{array},\left\{\begin{array}{l}
x_{2} \wedge y_{2} \text { if } x_{2} \vee y_{2}=1 \\
0 i f x_{2} \vee y_{2}<1
\end{array},\left\{\begin{array}{l}
x_{3} \vee y_{3} \text { if } x_{3} \wedge y_{3}=0 \\
1 \text { if } x_{3} \wedge y_{3} \neq 0
\end{array}\right)\right.\right.\right.
$$

6. $T_{06}(x, y)=\left(\max \left(0, x_{1}+y_{1}-1\right), \max \left(0, x_{2}+y_{2}-1\right), \min \left(1, x_{3}+y_{3}\right)\right)$.

7. $T_{07}(x, y)=\left(\max \left(0, x_{1}+y_{1}-1\right), \max \left(0, x_{2}+y_{2}-1\right), x_{3}+y_{3}-x_{3} y_{3}\right)$.

8. $T_{08}(x, y)=$

$$
\left(\max \left\{\frac{1}{2}\left(x_{1}+y_{1}-1+x_{1} y_{1}\right), 0\right\}, \max \left\{\frac{1}{2}\left(x_{2}+y_{2}-1+x_{2} y_{2}\right), 0\right\}, x_{3}+y_{3}-x_{3} y_{3}\right) \text {. }
$$

9. $T_{09}(x, y)=\left(x_{1} y_{1}, \max \left(0, x_{2}+y_{2}-1\right), x_{3}+y_{3}-x_{3} y_{3}\right)$.

10. $T_{010}(x, y)=\left(\max \left(0, x_{1}+y_{1}-1\right), x_{2} y_{2}, x_{3}+y_{3}-x_{3} y_{3}\right)$.

In this part we give some detailed proofs of picture $t$ - norms.

Proposition 3.7. Let $x, y \in D^{*}, x=\left(x_{1}, x_{2}, x_{3}\right), y=\left(y_{1}, y_{2}, y_{3}\right)$.

The mapping $T_{\min }$ is a picture fuzzy t-norm.

Proof. Let

$$
\begin{gathered}
x, y \in D^{*}, \text { then } x_{1}+x_{2} \leq 1-x_{3}, \text { and } y_{1}+y_{2} \leq 1-y_{3}, \\
\left(x_{1} \wedge y\right)+\left(x_{2} \wedge y_{2}\right) \leq \min \left(1-x_{3}, 1-y_{3}\right)=1-\max \left(x_{3}, y_{3}\right), \\
\left(x_{1} \wedge y\right)+\left(x_{2} \wedge y_{2}\right)+\max \left(x_{3}, y_{3}\right) \leq 1, \\
T_{\min }(x, y)=\left(\left(x_{1} \wedge y\right),\left(x_{2} \wedge y_{2}\right), \max \left(x_{3}, y_{3}\right)\right) \in D^{*} .
\end{gathered}
$$

The mapping $T_{\min }$ is a picture fuzzy t-norm, since other conditions easily are verified.

Let $x, y \in D^{*}, x=\left(x_{1}, x_{2}, x_{3}\right), y=\left(y_{1}, y_{2}, y_{3}\right)$.

Proposition 3.8. The mapping $T_{02}(x, y)=\left(\min \left(x_{1}, y_{1}\right), x_{2} y_{2}, \max \left(x_{3}, y_{3}\right)\right)$ is a picture fuzzy t-norm.

Proof. We remark that $x_{2} \cdot y_{2} \leq x_{2} \wedge y_{2} \Rightarrow\left(\left(x_{1} \wedge y_{1}\right)+x_{2} \cdot y_{2}+\max \left(x_{3}, y_{3}\right)\right) \leq T(x, y) \in D^{*}$. It implies that the mapping $T_{02}(x, y)=\left(\min \left(x_{1}, y_{1}\right), x_{2} y_{2}, \max \left(x_{3}, y_{3}\right)\right)$ is a picture fuzzy t-norm.

Proposition 3.9. The mapping $T_{04}(x, y)=\left(x_{1} y_{1}, x_{2} y_{2}, x_{3}+y_{3}-x_{3} y_{3}\right)$ is a picture fuzzy t-norm. 
Proof. We have

$$
\begin{aligned}
& x_{1} y_{1}+x_{2} y_{2} \leq\left(1-x_{2}-x_{3}\right)\left(1-y_{2}-y_{3}\right)+x_{2} y_{2} \\
&=\left(1-y_{2}-y_{3}-x_{2}+x_{2} y_{2}+x_{2} y_{3}-x_{3}+x_{3} y_{2}+x_{3} y_{3}\right)+x_{2} y_{2} \\
&=\left(1-x_{3}-y_{3}+x_{3} y_{3}\right)+\left(x_{2} y_{2}+x_{2} y_{3}+x_{2} y_{2}+x_{3} y_{2}-x_{2}-y_{2}\right) \\
&=\left(1-x_{3}-y_{3}+x_{3} y_{3}\right)+\left(x_{2}\left(y_{2}+y_{3}-1\right)+y_{2}\left(x_{2}+x_{3}-1\right)\right) \\
& \leq 1-x_{3}-y_{3}+x_{3} y_{3} \\
& \Rightarrow x_{1} y_{1}+x_{2} y_{2}+x_{3}+y_{3}-x_{3} y_{3} \leq 1 .
\end{aligned}
$$

Proposition 3.10. Let mapping $t_{2}$ is a fuzzy t-norm on $[0,1]$, then the mapping

$$
T_{0 t_{2}}(x, y)=\left(\min \left(x_{1}, y_{1}\right), t_{2}\left(x_{2}, y_{2}\right), \max \left(x_{3}, y_{3}\right)\right),
$$

is a picture fuzzy $t$ - norm.

Proof. See the proof of the Proposition 3.8.

Definition 3.11. A mapping $S: D^{*} \times D^{*} \rightarrow D^{*}$ is a picture fuzzy $t$-conorm if $S$ satisfies all of the following conditions

1. $S(x, y)=S(y, x), \forall x, y \in D^{*}$ (commutative),

2. $S(x, S(y, z))=S(S(x, y), z), \forall x, y, z \in D^{*}$ (associativity),

3. $S(x, y) \leq{ }_{1} S(x, z), \forall x, y, z \in D^{*}, y \leq{ }_{1} z$ (monotonicity),

4. $S\left(0_{D^{*}}, x\right) \in I(x), \forall x \in D^{*}$ (boundary condition).

Definition 3.12. A picture fuzzy t-conorm $S$ is called representable iff there exist two fuzzy t-norms $t_{1}, t_{2}$ on $[0,1]$ and a fuzzy t-conorm $s_{3}$ on $[0,1]$ satisfying.

Some examples of representable picture fuzzy t-conorms, for all $x, y \in D^{*}$.

1. $S_{\max }(x, y)=\left(\max \left(x_{1}, y_{1}\right), \min \left(x_{2}, y_{2}\right), \min \left(x_{3}, y_{3}\right)\right)$.

2. $S_{02}(x, y)=\left(\max \left(x_{1}, y_{1}\right), x_{2} y_{2}, \min \left(x_{3}, y_{3}\right)\right)$.

3. $S_{03}(x, y)=\left(\max \left(x_{1}, y_{1}\right), x_{2} y_{2}, x_{3} y_{3}\right)$.

4. $S_{04}(x, y)=\left(x_{1}+y_{1}-x_{1} y_{1}, x_{2} y_{2}, x_{3} y_{3}\right)$.

5. $S_{05}(x, y)=\left(x_{1} \vee y_{1},\left\{\begin{array}{l}x_{2} \wedge y_{2} \text { if } x_{2} \vee y_{2}=1 \\ 0 \text { if } x_{2} \vee y_{2}<1\end{array}, x_{3} \wedge y_{3}\right)\right.$.

6. $S_{06}(x, y)=\left(\left\{\begin{array}{l}x_{1} \vee y_{1} \text { if } x_{1} \wedge y_{1}=0 \\ 1 \text { if } x_{1} \wedge y_{1} \neq 0\end{array}, x_{2} \wedge y_{2},\left\{\begin{array}{l}x_{3} \wedge y_{3} \text { if } x_{3} \vee y_{3}=1 \\ 0 \text { if } x_{3} \vee y_{3}<1\end{array}\right)\right.\right.$. 
Proposition 3.13. For any representable picture fuzzy t-norm $T$ we have

$$
T_{05}(x, y) \leq_{1} T(x, y) \leq_{1} T_{\min }(x, y), \forall x, y \in D^{*} .
$$

Proposition 3.14. For any representable picture fuzzy t-conorm $S$ we have

$$
S_{05}(x, y) \leq_{1} S(x, y) \leq_{1} S_{06}(x, y), \forall x, y \in D^{*} .
$$

Proposition 3.15. Assume $T(u, v)$ is a $t$-representable intuitionistic fuzzy $t$-norm

$$
T(u, v)=\left(t_{1}\left(u_{1}, v_{1}\right), s_{3}\left(u_{3}, v_{3}\right)\right), \forall u=\left(u_{1}, u_{3}\right), v=\left(v_{1}, v_{3}\right) \in L^{*}
$$

where, $t_{1}$ is a fuzzy $t$-norm on $[0,1], s_{3}$ is a fuzzy t-conorm on $[0,1]$. Assume $t_{2}$ is a t-norm on $[0,1]$ satisfying.

$$
0 \leq t_{1}\left(x_{1}, y_{1}\right)+t_{2}\left(x_{2}, y_{2}\right)+s_{3}\left(x_{3}, y_{3}\right) \leq 1, \forall x, y \in D^{*}
$$

then

$$
T(x, y)=\left(t_{1}\left(x_{1}, y_{1}\right), t_{2}\left(x_{2}, y_{2}\right), s_{3}\left(x_{3}, y_{3}\right)\right), \forall x, y \in D^{*}
$$

is a representable picture fuzzy t-norm.

Proposition 3.16. Assume $S(u, v)$ is a t-representable intuitionistic fuzzy t-conorm

$$
S(u, v)=\left(s_{3}\left(u_{1}, v_{1}\right), t_{1}\left(u_{3}, v_{3}\right)\right), \quad \forall u=\left(u_{1}, u_{3}\right), v=\left(v_{1}, v_{3}\right) \in L^{*}
$$

where, $t_{1}$ is a fuzzy $t$-norm on $[0,1], s_{3}$ is a fuzzy t-conorm on $[0,1]$. Assume $t_{2}$ is a $t$-norm on $[0,1]$ satisfies $0 \leq t_{1}\left(x_{1}, y_{1}\right)+t_{2}\left(x_{2}, y_{2}\right)+s_{3}\left(x_{3}, y_{3}\right) \leq 1, \forall x, y \in D^{*}$ then

$$
S(x, y)=\left(s_{3}\left(x_{1}, y_{1}\right), t_{2}\left(x_{2}, y_{2}\right), t_{1}\left(x_{3}, y_{3}\right)\right), \forall x, y \in D^{*}
$$

is a representable picture fuzzy t-conorm.

Now we define some new concepts for the picture fuzzy logic.

Definition 3.17. A picture fuzzy t-norm $T$ is called Achimerdean iff

$$
\forall x \in D^{*} \backslash\left\{0_{D^{*}}, 1_{D^{*}}\right\}, T(x, x)<_{1} x .
$$

Definition 3.18. A picture fuzzy t-norm $T$ is called

1. Nilpotent iff: $\exists x, y \in D^{*} \backslash\left\{0_{D^{*}}\right\}, T(x, y)=0_{D^{*}}$.

2. Strict iff: $\forall x, y \in D^{*} \backslash\left\{0_{D^{*}}\right\}, T(x, y) \neq 0_{D^{*}}$.

With these defitions we have the following propositions.

Proposition 3.19. Let

then $T^{*} \cap T^{* *}=\emptyset$.

$T^{*}=\{$ Nilpotent picture $t-$ norms $\}$,

$T^{* *}=\{$ strict picture $t-$ norms $\}$

Definition 3.20. A picture fuzzy t-conorm $S$ is called Achimerdean iff

$$
\forall x \in D^{*} \backslash\left\{0_{D^{*}}, 1_{D^{*}}\right\}, S(x, x)>_{1} x .
$$

Definition 3.21. A picture fuzzy t-conorm $S$ is called 
1. Nilpotent iff: $\exists x, y \in D^{*} \backslash\left\{1_{D^{*}}\right\}, S(x, y)=1_{D^{*}}$.

2. Strict iff: $\forall x, y \in D^{*} \backslash\left\{1_{D^{*}}\right\}, S(x, y) \neq 1_{D^{*}}$.

Proposition 3.22. Let

$S^{*}=\{$ nilpotent picture fuzzy $t-$ conorms $\}$,

$S^{* *}=\{$ strict picture $t-$ conorms $\}$

then $S^{*} \cap S^{* *}=\emptyset$.

Proposition 3.23. Assume $T$ is a representable picture fuzzy t-norm

$$
T(x, y)=\left(t_{1}\left(x_{1}, y_{1}\right), t_{2}\left(x_{2}, y_{2}\right), s_{3}\left(x_{3}, y_{3}\right)\right), \forall x, y \in D^{*},
$$

and $t_{1}, t_{2}, s_{3}$ are Archimedean on $[0,1]$, then $T$ is Archimedean.

Proof. For all $x \in D^{*} \backslash\left\{0_{D^{*}}, 1_{D^{*}}\right\}$, we have

$$
T(x, x)=\left(t_{1}\left(x_{1}, x_{1}\right), t_{2}\left(x_{2}, x_{2}\right), s_{3}\left(x_{3}, x_{3}\right)\right) .
$$

Since $t_{1}, t_{2}, s_{3}$ are Archimedean on [0,1]. It follows that $t_{1}\left(x_{1}, x_{1}\right)<x_{1}, s_{3}\left(x_{3}, x_{3}\right)>x_{3}$, so $T(x, x)<_{1} x$. Thus $T$ is Archimedean.

Proposition 3.24. Assume $S$ is a representable picture fuzzy t-conorm

$$
S(x, y)=\left(s_{3}\left(x_{1}, y_{1}\right), t_{2}\left(x_{2}, y_{2}\right), t_{1}\left(x_{3}, y_{3}\right)\right), \forall x, y \in D^{*} .
$$

and $t_{1}, t_{2}, s_{3}$ are Archimedean on $[0,1]$, then $S$ is Archimedean.

\section{SOME SUBCLASSES OF REPRESENTABLE PICTURE FUZZY T-NORMS}

We can give some subclasses of representable picture fuzzy t-norms.

\subsection{Strict-strict-strict t-norms subclass, denoted by $\Delta_{s s s}$}

Definition 4.1. A picture fuzzy t-norm $T$ is called strict-strict-strict iff

$$
T(x, y)=\left(t_{1}\left(x_{1}, y_{1}\right), t_{2}\left(x_{2}, y_{2}\right), s_{3}\left(x_{3}, y_{3}\right)\right), \forall x, y \in D^{*},
$$

where $t_{1}, t_{2}$ are strict fuzzy t-norms on $[0,1]$ and $s_{3}$ is a strict fuzzy t-conorm on $[0,1]$.

Example 4.1. $T_{1}(x, y)=\left(x_{1} y_{1}, x_{2} y_{2}, x_{3}+y_{3}-x_{3} y_{3}\right)$,

$$
\begin{aligned}
& T_{2}(x, y)=\left(\frac{x_{1} y_{1}}{\lambda_{1}+\left(1-\lambda_{1}\right)\left(x_{1}+y_{1}-x_{1} y_{1}\right)}, \frac{x_{2} y_{2}}{\lambda_{2}+\left(1-\lambda_{2}\right)\left(x_{2}+y_{2}-x_{2} y_{2}\right)},\left(x_{3}^{a}+y_{3}^{a}-x_{3}^{a} y_{3}^{a}\right)^{\frac{1}{a}}\right), \\
& \lambda_{1}, \lambda_{2}, a \in[1,+\infty)
\end{aligned}
$$




\subsection{Nipoltent-nipoltent-nipoltent t-norms subclass, denoted by $\Delta_{n n n}$}

Definition 4.2. A picture fuzzy t-norm $T$ is called nipoltent-nipoltent-nipoltent iff

$$
T(x, y)=\left(t_{1}\left(x_{1}, y_{1}\right), t_{2}\left(x_{2}, y_{2}\right), s_{3}\left(x_{3}, y_{3}\right)\right), \forall x, y \in D^{*}
$$

where $t_{1}, t_{2}$ are nipoltent fuzzy t-norms on $[0,1]$ and $s_{3}$ is a nipoltent fuzzy t-conorm on $[0,1]$.

\section{Examples 4.2.}

$$
\begin{gathered}
T_{3}(x, y)=\left(0 \vee\left(x_{1}+y_{1}-1\right), 0 \vee\left(x_{2}+y_{2}-1\right), 1 \wedge\left(x_{3}+y_{3}\right)\right), \\
T_{4}(x, y)=\left(\left(\left(x_{1}+y_{1}-1\right)\left(1+\lambda_{1}\right)-\lambda_{1} x_{1} y_{1}\right) \vee 0,\left(\left(x_{2}+y_{2}-1\right)\left(1+\lambda_{2}\right)-\lambda_{2} x_{2} y_{2}\right) \vee 0,\right. \\
\left.1 \wedge\left(x_{3}^{a}+y_{3}^{a}\right)^{\frac{1}{a}}\right), \lambda_{1}, \lambda_{2} \in[0,+\infty), a \geq 1, \\
T_{5}(x, y)=\left(\left(0 \vee\left(x_{1}^{a}+y_{1}^{a}-1\right)\right) \frac{1}{a},\left(0 \vee\left(x_{2}^{b}+y_{2}^{b}-1\right)\right) \frac{1}{b}, 1 \wedge\left(x_{3}^{c}+y_{3}^{c}\right)^{\frac{1}{c}}\right), a, b, c \geq 1, \\
T_{6}(x, y)=\left(\left(\frac{1}{a}\left(x_{1}+y_{1}-1+(a-1) x_{1} y_{1}\right) \vee 0\right),\left(\frac{1}{b}\left(x_{2}+y_{2}-1+(b-1) x_{2} y_{2}\right) \vee 0\right),\right. \\
\left.1 \wedge\left(x_{3}^{c}+y_{3}^{c}\right) \frac{1}{c}\right), a, b \in(0,1] ; c \geq 1, \\
T_{7}(x, y)=\left(\left(\frac{1}{a}\left(x_{1}+y_{1}-1+(a-1) x_{1} y_{1}\right) \vee 0\right),\left(\left(x_{2}+y_{2}-1\right)(1+\lambda)-\lambda x_{2} y_{2}\right) \vee 0,\right. \\
\left.1 \wedge\left(x_{3}^{b}+y_{3}^{b}\right)^{\frac{1}{b}}\right), a \in(0,1], \lambda \geq 0, b \geq 1, \\
T_{8}(x, y)=\left(\left(\left(x_{1}+y_{1}-1\right)(1+\lambda)-\lambda x_{1} y_{1}\right) \vee 0,\left(\frac{1}{a}\left(x_{2}+y_{2}-1+(a-1) x_{2} y_{2}\right) \vee 0\right),\right. \\
\left.1 \wedge\left(x_{3}^{b}+y_{3}^{b}\right) \frac{1}{b}\right), a \in(0,1], b \geq 1, \lambda \geq 0, \\
T_{11}(x, y)=\left(\left(\left(x_{1}+y_{1}-1\right)(1+\lambda)-\lambda x_{1} y_{1}\right) \vee 0,0 \vee\left(x_{2}^{a}+y_{2}^{a}-1\right)^{\frac{1}{a}},\right. \\
\left.1 \wedge\left(x_{3}^{b}+y_{3}^{b}\right)^{\frac{1}{b}}\right), \lambda \geq 0, a, b \geq 1, \\
T_{10}(x, y)=\left(0 \vee\left(x_{1}^{a}+y_{1}^{a}-1\right)^{\frac{1}{a}},\left(\frac{1}{b}\left(x_{2}+y_{2}-1+(b-1) x_{2} y_{2}\right) \vee 0\right),\right. \\
\left.1 \wedge\left(x_{3}^{c}+y_{3}^{c}\right)^{\frac{1}{c}}\right), b \in(0,1], a, c \geq 1, \\
\left.1 \wedge\left(x_{3}^{b}+y_{3}^{b}\right)^{\frac{1}{b}}\right), \lambda \geq 0, a, b \geq 1 . \\
\left.1 \wedge\left(x_{3}^{c}+y_{3}^{c}\right)^{\frac{1}{c}}\right), a \in(0,1], b, c \geq 1, \\
\end{gathered}
$$




\subsection{Nipoltent-nipoltent-strict t-norms subclass, denoted by $\Delta_{n n s}$}

Definition 4.3. A picture fuzzy t-norm $T$ is called nipoltent-nipoltent-strict iff

$$
T(x, y)=\left(t_{1}\left(x_{1}, y_{1}\right), t_{2}\left(x_{2}, y_{2}\right), s_{3}\left(x_{3}, y_{3}\right)\right), \forall x, y \in D^{*},
$$

where $t_{1}, t_{2}$ are nipoltent fuzzy t-norms on $[0,1]$ and $s_{3}$ is a strict fuzzy t-conorm on $[0,1]$.

\section{Examples 4.3.}

$$
\begin{gathered}
T_{13}(x, y)=\left(0 \vee\left(x_{1}+y_{1}-1\right), 0 \vee\left(x_{2}+y_{2}-1\right), x_{3}+y_{3}-x_{3} y_{3}\right), \\
T_{14}(x, y)=\left(\frac{1}{2}\left(x_{1}+y_{1}-1+x_{1} y_{1}\right) \vee 0, \frac{1}{2}\left(x_{2}+y_{2}-1+x_{2} y_{2}\right) \vee 0,\left(x_{3}^{a}+y_{3}^{a}-x_{3}^{a} y_{3}^{a}\right)^{\frac{1}{a}}\right),
\end{gathered}
$$

$a \geq 1$.

$$
\begin{aligned}
& T_{15}(x, y)=\left(\left(\left(x_{1}+y_{1}-1\right)\left(1+\lambda_{1}\right)-\lambda_{1} x_{1} y_{1}\right) \vee 0,\left(\left(x_{2}+y_{2}-1\right)\left(1+\lambda_{2}\right)-\lambda_{2} x_{2} y_{2}\right) \vee 0,\right. \\
& \left.\left(x_{3}^{a}+y_{3}^{a}-x_{3}^{a} y_{3}^{a}\right)^{\frac{1}{a}}\right), \lambda_{1}, \lambda_{2} \in[0,+\infty), a \geq 1, \\
& T_{16}(x, y)=\left(0 \vee\left(x_{1}^{a}+y_{1}^{a}-1\right)^{\frac{1}{a}},\left(x_{2}^{b}+y_{2}^{b}-1\right)^{\frac{1}{b}} \vee 0,\left(x_{3}^{c}+y_{3}^{c}-x_{3}^{c} y_{3}^{c}\right)^{\frac{1}{c}}\right), a, b, c \geq 1, \\
& T_{17}(x, y)=\left(\frac{1}{a}\left(x_{1}+y_{1}-1+(a-1) x_{1} y_{1}\right) \vee 0,0 \vee\left(x_{2}^{b}+y_{2}^{b}-1\right)^{\frac{1}{b}},\left(x_{3}^{c}+y_{3}^{c}-x_{3}^{c} y_{3}^{c}\right)^{\frac{1}{c}}\right), \\
& a \in(0,1] ; b, c \geq 1, \\
& T_{18}(x, y)=\left(\frac{1}{a}\left(x_{1}+y_{1}-1+(a-1) x_{1} y_{1}\right) \vee 0, \frac{1}{b}\left(x_{2}+y_{2}-1+(b-1) x_{2} y_{2}\right) \vee 0,\right. \\
& \left.\left(x_{3}^{c}+y_{3}^{c}-x_{3}^{c} y_{3}^{c}\right)^{\frac{1}{c}}\right), a, b \in(0,1] ; c \geq 1 \text {, } \\
& T_{19}(x, y)=\left(\frac{1}{a}\left(x_{1}+y_{1}-1+(a-1) x_{1} y_{1}\right) \vee 0,\left(\left(x_{2}+y_{2}-1\right)(1+b)-b x_{2} y_{2}\right) \vee 0,\right. \\
& \left.\left(x_{3}^{c}+y_{3}^{c}-x_{3}^{c} y_{3}^{c}\right)^{\frac{1}{c}}\right), a \in(0,1] ; b \geq 0 ; c \geq 1, \\
& T_{20}(x, y)=\left(0 \vee\left(x_{1}^{a}+y_{1}^{a}-1\right)^{\frac{1}{a}}, \frac{1}{b}\left(x_{2}+y_{2}-1+(b-1) x_{2} y_{2}\right) \vee 0,\right. \\
& \left.\left(x_{3}^{c}+y_{3}^{c}-x_{3}^{c} y_{3}^{c}\right)^{\frac{1}{c}}\right), b \in(0,1] ; a, c \geq 1, \\
& T_{21}(x, y)=\left(\left(\left(x_{1}+y_{1}-1\right)(1+a)-a x_{1} y_{1}\right) \vee 0, \frac{1}{b}\left(x_{2}+y_{2}-1+(b-1) x_{2} y_{2}\right) \vee 0,\right. \\
& \left.\left(x_{3}^{c}+y_{3}^{c}-x_{3}^{c} y_{3}^{c}\right)^{\frac{1}{c}}\right), a \geq 0 ; b \in(0,1] ; c \geq 1, \\
& T_{22}(x, y)=\left(\left(\left(x_{1}+y_{1}-1\right)(1+\lambda)-\lambda x_{1} y_{1}\right) \vee 0,0 \vee\left(x_{2}^{a}+y_{2}^{a}-1\right)^{\frac{1}{a}},\right. \\
& \left.\left(x_{3}^{b}+y_{3}^{b}-x_{3}^{b} y_{3}^{b}\right)^{\frac{1}{b}}\right), \lambda \geq 0, a, b \geq 1, \\
& T_{23}(x, y)=\left(0 \vee\left(x_{1}^{a}+y_{1}^{a}-1\right)^{\frac{1}{a}},\left(\left(x_{2}+y_{2}-1\right)(1+\lambda)-\lambda x_{2} y_{2}\right) \vee 0,\right. \\
& \left.\left(x_{3}^{b}+y_{3}^{b}-x_{3}^{b} y_{3}^{b}\right)^{\frac{1}{b}}\right), \lambda \geq 0, a, b \geq 1 .
\end{aligned}
$$




\subsection{Strict-nipoltent-strict t-norms subclass, denoted by $\Delta_{\text {sns }}$}

Definition 4.4. A picture fuzzy t-norm $T$ is called strict-nipoltent-strict iff

$$
T(x, y)=\left(t_{1}\left(x_{1}, y_{1}\right), t_{2}\left(x_{2}, y_{2}\right), s_{3}\left(x_{3}, y_{3}\right)\right), \forall x, y \in D^{*},
$$

where $t_{1}$ is a strict fuzzy t-norm on $[0,1], t_{2}$ is a nipoltent fuzzy t-norm on $[0,1]$ and $s_{3}$ is a strict fuzzy t-conorm on $[0,1]$.

\section{Example 4.4.}

$$
\begin{gathered}
T_{24}(x, y)=\left(x_{1} y_{1}, 0 \vee\left(x_{2}+y_{2}-1\right), x_{3}+y_{3}-x_{3} y_{3}\right) \\
T_{25}(x, y)=\left(\frac{x_{1} y_{1}}{\lambda_{1}+\left(1-\lambda_{1}\right)\left(x_{1}+y_{1}-x_{1} y_{1}\right)},\left(\left(x_{2}+y_{2}-1\right)\left(1+\lambda_{2}\right)-\lambda_{2} x_{2} y_{2}\right) \vee 0,\right. \\
\left.\left(x_{3}^{a}+y_{3}^{a}-x_{3}^{a} y_{3}^{a}\right)^{\frac{1}{a}}\right), \lambda_{1} \geq 1, \lambda_{2} \geq 0, a \geq 1, \\
T_{26}(x, y)=\left(\frac{x_{1} y_{1}}{\lambda_{1}+\left(1-\lambda_{1}\right)\left(x_{1}+y_{1}-x_{1} y_{1}\right)}, 0 \vee\left(x_{2}^{a}+y_{2}^{a}-1\right) \frac{1}{a},\right. \\
T_{27}(x, y)=\left(\frac{\left.\left(x_{3}^{b}+y_{3}^{b}-x_{3}^{b} y_{3}^{b}\right)^{\frac{1}{b}}\right), \lambda_{1} \geq 1, a, b \geq 1,}{\lambda_{1}+\left(1-\lambda_{1}\right)\left(x_{1}+y_{1}-x_{1} y_{1}\right)}, \frac{1}{a}\left(x_{2}+y_{2}-1+(a-1) x_{2} y_{2}\right) \vee 0,\right. \\
\left.\left(x_{3}^{b}+y_{3}^{b}-x_{3}^{b} y_{3}^{b}\right)^{\frac{1}{b}}\right), \lambda_{1}, b \geq 1, a \in(0,1] .
\end{gathered}
$$

\subsection{Nipoltent-strict-strict t-norms subclass, denoted by $\Delta_{n s s}$}

Definition 4.5. A picture fuzzy t-norm $T$ is called nipoltent-strict-strict iff

$$
T(x, y)=\left(t_{1}\left(x_{1}, y_{1}\right), t_{2}\left(x_{2}, y_{2}\right), s_{3}\left(x_{3}, y_{3}\right)\right), \forall x, y \in D^{*},
$$

where $t_{1}$ is a nipoltent fuzzy t-norm on $[0,1], t_{2}$ is a strict fuzzy t-norm on $[0,1]$ and $s_{3}$ is a strict fuzzy t-conorm on $[0,1]$.

\section{Example 4.5.}

$$
\begin{gathered}
T_{28}(x, y)=\left(0 \vee\left(x_{1}+y_{1}-1\right), x_{2} y_{2}, x_{3}+y_{3}-x_{3} y_{3}\right), \\
T_{29}(x, y)=\left(\frac{1}{a}\left(x_{1}+y_{1}-1+(a-1) x_{1} y_{1}\right) \vee 0, \frac{x_{2} y_{2}}{\lambda+(1-\lambda)\left(x_{2}+y_{2}-x_{2} y_{2}\right)},\right. \\
\left.\left(x_{3}^{b}+y_{3}^{b}-x_{3}^{b} y_{3}^{b}\right)^{\frac{1}{b}}\right), a \in(0,1] ; b, \lambda \geq 1, \\
T_{30}(x, y)=\left(0 \vee\left(x_{1}^{a}+y_{1}^{a}-1\right)^{\frac{1}{a}}, \frac{x_{2} y_{2}}{\lambda+(1-\lambda)\left(x_{2}+y_{2}-x_{2} y_{2}\right)},\right. \\
\left.\left(x_{3}^{b}+y_{3}^{b}-x_{3}^{b} y_{3}^{b}\right)^{\frac{1}{b}}\right), a, b, \lambda \geq 1, \\
T_{31}(x, y)=\left(\left(\left(x_{1}+y_{1}-1\right)\left(1+\lambda_{1}\right)-\lambda_{1} x_{1} y_{1}, \frac{x_{2} y_{2}}{\lambda_{2}+\left(1-\lambda_{2}\right)\left(x_{2}+y_{2}-x_{2} y_{2}\right)},\right.\right. \\
\left.\left(x_{3}^{a}+y_{3}^{a}-x_{3}^{a} y_{3}^{a}\right)^{\frac{1}{a}}\right), a, \lambda_{2} \geq 1, \lambda_{1} \in(0,1] .
\end{gathered}
$$

Proposition 4.6. There doesn't exist representable picture fuzzy t-norm $T$ 


$$
T(x, y)=\left(t_{1}\left(x_{1}, y_{1}\right), t_{2}\left(x_{2}, y_{2}\right), s_{3}\left(x_{3}, y_{3}\right)\right), \forall x, y \in D^{*},
$$

where $t_{1}$ or $t_{2}$ is a strict fuzzy t-norm on $[0,1]$, and $s_{3}$ is a nipoltent fuzzy $t$-conorm on $[0,1]$.

Proof. Assume $T(x, y)=\left(t_{1}\left(x_{1}, y_{1}\right), t_{2}\left(x_{2}, y_{2}\right), s_{3}\left(x_{3}, y_{3}\right)\right), \forall x, y \in D^{*}$, with $t_{1}$ is a strict t-norm and there exist $x_{3}, y_{3} \in(0,1)$ such that $S_{3}\left(x_{3}, y_{3}\right)=1$. Let $x_{1}, x_{2} \neq 0 \mid x_{1}+x_{2}+x_{3} \leq$ $1 ; y_{1}, y_{2} \neq 0 \mid y_{1}+y_{2}+y_{3} \leq 1$, and since $t_{1}$ is strict t-norm then $t_{1}\left(x_{1}, y_{1}\right)>0$.

Let $x=\left(x_{1}, x_{2}, x_{3}\right), y=\left(y_{1}, y_{2}, y_{3}\right)$, we have a contradiction $t_{1}\left(x_{1}, y_{1}\right)+t_{2}\left(x_{2}, y_{2}\right)+$ $s_{3}\left(x_{3}, y_{3}\right)>1$.

Similarly, if $t_{2}$ is strict t-norm and $s_{3}$ is nipoltent t-conorm then we have a contradiction.

Proposition 4.7. If $T$ belongs to one of four classes $\Delta_{\text {sss }}, \Delta_{n n s}, \Delta_{\text {sns }}, \Delta_{n s s}$ then $T$ is strict.

Proof. Assume for all $x, y \in D^{*}, s_{3}$ is a strict fuzzy t-conorm on [0,1], $T$ is a representable picture fuzzy t-norm $T(x, y)=\left(t_{1}\left(x_{1}, y_{1}\right), t_{2}\left(x_{2}, y_{2}\right), s_{3}\left(x_{3}, y_{3}\right)\right)$ and $T$ is nipoltent.

Then $\exists x, y \in D^{*} \backslash\left\{0_{D^{*}}\right\}, T(x, y)=0_{D^{*}}$, and it implies $t_{1}\left(x_{1}, y_{1}\right)=0, t_{2}\left(x_{2}, y_{2}\right)=$ $0, s_{3}\left(x_{3}, y_{3}\right)=1$. Since $s_{3}$ is a strict fuzzy t-conorm on $[0,1]$, then $x_{3}=1$ or $y_{3}=1$, which is a contradiction.

Proposition 4.8. If $T$ belongs to the class $\Delta_{n n n}$ then $T$ is a nipoltent picture fuzzy t-norm.

Proof. Assume $T \in \Delta_{n n n}, \forall x, y \in D^{*}: T(x, y)=\left(t_{1}\left(x_{1}, y_{1}\right), t_{2}\left(x_{2}, y_{2}\right), s_{3}\left(x_{3}, y_{3}\right)\right)$.

Since $t_{1}, t_{2}$ are nipoltent fuzzy t-norms on $[0,1]$, we have

$\exists x_{1}, y_{1}, x_{2}, y_{2} \mid t_{1}\left(x_{1}, y_{1}\right)=0, t_{2}\left(x_{2}, y_{2}\right)=0$. Since $t_{1}, t_{2}$ are not decreasing, so $\forall x_{1}^{\prime} \leq$ $x_{1}, y_{1}^{\prime} \leq y_{1} ; x_{2}^{\prime} \leq x_{2}, y_{2}^{\prime} \leq y_{2} \mid t_{1}\left(x_{1}^{\prime}, y_{1}^{\prime}\right)=0, t_{2}\left(x_{2}^{\prime}, y_{2}^{\prime}\right)=0$. Since $s$ is a nipoltent fuzzy t-conorm on $[0,1]$ so $\exists x_{3}, y_{3} \neq 1 \mid s_{3}\left(x_{3}, y_{3}\right)=1$. Let $x=\left(x_{1}^{\prime}, x_{2}^{\prime}, x_{3}\right), y=\left(y_{1}^{\prime}, y_{2}^{\prime}, y_{3}\right) \in D^{*}$. Then $T(x, y)=\left(t_{1}\left(x_{1}^{\prime}, y_{1}^{\prime}\right), t_{2}\left(x_{2}^{\prime}, y_{2}^{\prime}\right), s_{3}\left(x_{3}, y_{3}\right)\right)=0_{D^{*}}$. $T$ is a nipoltent picture fuzzy t-norm.

\section{SOME SUBCLASSES OF REPRESENTABLE PICTURE FUZZY T-CONORMS}

Similarly to the Section 4, we can give some subclasses of representable picture fuzzy t-conorms.

\subsection{Strict-strict-strict t-conorms subclass, denoted by $\nabla_{s s s}$}

Definition 5.1. A picture fuzzy t-conorm $S$ is called strict-strict-strict iff

$$
S(x, y)=\left(s_{3}\left(x_{1}, y_{1}\right), t_{2}\left(x_{2}, y_{2}\right), t_{1}\left(x_{3}, y_{3}\right)\right), \forall x, y \in D^{*} .
$$

where $t_{1}, t_{2}$ are strict fuzzy t-norms on $[0,1]$ and $s_{3}$ is a strict fuzzy t-conorm on $[0,1]$.

\section{Examples 5.1.}

$$
S_{1}(x, y)=\left(x_{1}+y_{1}-x_{1} y_{1}, x_{2} y_{2}, x_{3} y_{3}\right),
$$




$$
\begin{aligned}
& S_{2}(x, y) \\
& =\left(\left(x_{1}^{a}+y_{1}^{a}-x_{1}^{a} y_{1}^{a}\right)^{\frac{1}{a}}, \frac{x_{2} y_{2}}{\lambda_{1}+\left(1-\lambda_{1}\right)\left(x_{2}+y_{2}-x_{2} y_{2}\right)}, \frac{x_{3} y_{3}}{\lambda_{2}+\left(1-\lambda_{2}\right)\left(x_{3}+y_{3}-x_{3} y_{3}\right)}\right),
\end{aligned}
$$

with $\lambda_{1}, \lambda_{2}, a \in[1,+\infty)$.

\subsection{Nipoltent-nipoltent-nipoltent t-conorms subclass, denoted by $\nabla_{n n n}$}

Definition 5.2. A picture fuzzy t-conorm $S$ is called nipoltent-nipoltent-nipoltent iff

$$
S(x, y)=\left(s_{3}\left(x_{1}, y_{1}\right), t_{2}\left(x_{2}, y_{2}\right), t_{1}\left(x_{3}, y_{3}\right)\right), \forall x, y \in D^{*},
$$

where $t_{1}, t_{2}$ are nipoltent fuzzy t-norms on $[0,1]$ and $s_{3}$ is a nipoltent fuzzy t-conorm on $[0,1]$.

\section{Examples 5.2.}

$$
\begin{gathered}
S_{3}(x, y)=\left(1 \wedge\left(x_{1}+y_{1}\right), 0 \vee\left(x_{2}+y_{2}-1\right), 0 \vee\left(x_{3}+y_{3}-1\right)\right), \\
S_{4}(x, y)=\left(1 \wedge\left(x_{1}^{a}+y_{1}^{a}\right)^{\frac{1}{a}},\left(\left(x_{2}+y_{2}-1\right)\left(1+\lambda_{1}\right)-\lambda_{1} x_{2} y_{2}\right) \vee 0,\right. \\
\left.\left(\left(x_{3}+y_{3}-1\right)\left(1+\lambda_{2}\right)-\lambda_{2} x_{3} y_{3}\right) \vee 0\right), \lambda_{1}, \lambda_{2} \in[0,+\infty), a \geq 1, \\
S_{5}(x, y)=\left(1 \wedge\left(x_{1}^{a}+y_{1}^{a}\right)^{\frac{1}{a}},\left(0 \vee\left(x_{2}^{b}+y_{2}^{b}-1\right)\right)^{\frac{1}{b}}, 0 \vee\left(x_{3}^{c}+y_{3}^{c}-1\right)^{\frac{1}{c}}\right), \\
a, b, c \geq 1, \\
S_{6}(x, y)=\left(1 \wedge\left(x_{1}^{a}+y_{1}^{a}\right)^{\frac{1}{a}},\left(\frac{1}{b}\left(x_{2}+y_{2}-1+(b-1) x_{2} y_{2}\right) \vee 0\right),\right. \\
\left.\quad\left(\frac{1}{c}\left(x_{3}+y_{3}-1+(c-1) x_{3} y_{3}\right) \vee 0\right)\right), a \geq 1 ; b, c \in(0,1], \\
S_{7}(x, y)=\left(1 \wedge\left(x_{1}^{a}+y_{1}^{a}\right)^{\frac{1}{a}},\left(\frac{1}{b}\left(x_{2}+y_{2}-1+(b-1) x_{2} y_{2}\right) \vee 0\right),\right. \\
\left.\left(\left(x_{3}+y_{3}-1\right)(1+\lambda)-\lambda x_{3} y_{3}\right) \vee 0\right), a \geq 1, b \in(0,1], \lambda \geq 0, \\
S_{8}(x, y)=\left(1 \wedge\left(x_{1}^{a}+y_{1}^{a}\right)^{\frac{1}{a}},\left(\left(x_{2}+y_{2}-1\right)(1+\lambda)-\lambda x_{2} y_{2}\right) \vee 0,\right. \\
\left.\left(\frac{1}{b}\left(x_{3}+y_{3}-1+(b-1) x_{3} y_{3}\right) \vee 0\right)\right), a \geq 1, b \in(0,1], \lambda \geq 0, \\
S_{9}(x, y)=\left(1 \wedge\left(x_{1}^{a}+y_{1}^{a}\right)^{\frac{1}{a}},\left(\frac{1}{b}\left(x_{2}+y_{2}-1+(b-1) x_{2} y_{2}\right) \vee 0\right),\right. \\
\left.0 \vee\left(x_{3}^{c}+y_{3}^{c}-1\right)^{\frac{1}{c}}\right), b \in(0,1], a, c \geq 1, \\
S_{10}(x, y)=\left(1 \wedge\left(x_{1}^{a}+y_{1}^{a}\right)^{\frac{1}{a}}, 0 \vee\left(x_{2}^{b}+y_{2}^{b}-1\right)^{\frac{1}{b}},\right. \\
\left.\left(\frac{1}{c}\left(x_{3}+y_{3}-1+(c-1) x_{3} y_{3}\right) \vee 0\right)\right), c \in(0,1], a, b \geq 1, \\
S_{12}(x, y)=\left(1 \wedge\left(x_{1}^{a}+y_{1}^{a}\right)^{\frac{1}{a}}, 0 \vee\left(x_{2}^{b}+y_{2}^{b}-1\right)^{\frac{1}{b}},\right. \\
S_{11}(x, y)=\left(1 \wedge\left(x_{1}^{a}+y_{1}^{a}\right)^{\frac{1}{a}},\left(\left(x_{2}+y_{2}-1\right)(1+\lambda)-\lambda x_{3} y_{3}\right) \vee 0\right), \lambda \geq 0, a, b \geq 1 . \\
\left.0 \vee\left(x_{3}^{b}+y_{3}^{b}-1\right)^{\frac{1}{b}}\right), \lambda \geq 0, a, b \geq 1, \\
\left.x_{2} y_{2}\right) \vee 0,
\end{gathered}
$$




\subsection{Strict-nipoltent-nipoltent t-conorms subclass, denoted by $\nabla_{s n n}$}

Definition 5.3. A picture fuzzy t-conorm $S$ is called strict-nipoltent-nipoltent iff

$$
S(x, y)=\left(s_{3}\left(x_{1}, y_{1}\right), t_{2}\left(x_{2}, y_{2}\right), t_{1}\left(x_{3}, y_{3}\right)\right), \forall x, y \in D^{*},
$$

where $t_{1}, t_{2}$ are nipoltent fuzzy t-norms on $[0,1]$ and $s_{3}$ is a strict fuzzy t-conorm on $[0,1]$.

\section{Examples 5.3.}

$$
\begin{aligned}
& S_{13}(x, y)=\left(x_{1}+y_{1}-x_{1} y_{1}, 0 \vee\left(x_{2}+y_{2}-1\right), 0 \vee\left(x_{3}+y_{3}-1\right)\right), \\
& S_{14}(x, y)=\left(\left(x_{1}^{a}+y_{1}^{a}-x_{1}^{a} y_{1}^{a}\right)^{\frac{1}{a}}, \frac{1}{2}\left(x_{2}+y_{2}-1+x_{2} y_{2}\right) \vee 0,\right. \\
& \left.\frac{1}{2}\left(x_{3}+y_{3}-1+x_{3} y_{3}\right) \vee 0\right), a \geq 1, \\
& S_{15}(x, y)=\left(\left(x_{1}^{a}+y_{1}^{a}-x_{1}^{a} y_{1}^{a}\right)^{\frac{1}{a}},\left(\left(x_{2}+y_{2}-1\right)\left(1+\lambda_{1}\right)-\lambda_{1} x_{2} y_{2}\right) \vee 0,\right. \\
& \left.\left(\left(x_{3}+y_{3}-1\right)\left(1+\lambda_{2}\right)-\lambda_{2} x_{3} y_{3}\right) \vee 0\right), \lambda_{1}, \lambda_{2} \in[0,+\infty), a \geq 1, \\
& S_{16}(x, y)=\left(\left(x_{1}^{c}+y_{1}^{c}-x_{1}^{c} y_{1}^{c}\right)^{\frac{1}{c}}, 0 \vee\left(x_{2}^{a}+y_{2}^{a}-1\right)^{\frac{1}{a}}, 0 \vee\left(x_{3}^{b}+y_{3}^{b}-1\right)^{\frac{1}{b}}\right), a, b, c \geq 1, \\
& S_{17}(x, y)=\left(\left(x_{1}^{a}+y_{1}^{a}-x_{1}^{a} y_{1}^{a}\right)^{\frac{1}{a}}, \frac{1}{b}\left(x_{2}+y_{2}-1+(b-1) x_{2} y_{2}\right) \vee 0,\right. \\
& \left.0 \vee\left(x_{3}^{c}+y_{3}^{c}-1\right)^{\frac{1}{c}}\right), b \in(0,1] ; a, c \geq 1, \\
& S_{18}(x, y)=\left(\left(x_{1}^{a}+y_{1}^{a}-x_{1}^{a} y_{1}^{a}\right)^{\frac{1}{a}}, \frac{1}{b}\left(x_{2}+y_{2}-1+(b-1) x_{2} y_{2}\right) \vee 0,\right. \\
& \left.\frac{1}{c}\left(x_{3}+y_{3}-1+(c-1) x_{3} y_{3}\right) \vee 0\right), b, c \in(0,1] ; a \geq 1, \\
& S_{19}(x, y)=\left(\left(x_{1}^{a}+y_{1}^{a}-x_{1}^{a} y_{1}^{a}\right)^{\frac{1}{a}}, \frac{1}{b}\left(x_{2}+y_{2}-1+(b-1) x_{2} y_{2}\right) \vee 0,\right. \\
& \left.\left(\left(x_{3}+y_{3}-1\right)(1+c)-c x_{3} y_{3}\right) \vee 0\right), a \geq 1, b \in(0,1] ; c \geq 0, \\
& S_{20}(x, y)=\left(\left(x_{1}^{a}+y_{1}^{a}-x_{1}^{a} y_{1}^{a}\right)^{\frac{1}{a}},\left(x_{2}^{b}+y_{2}^{b}-1\right)^{\frac{1}{b}} \vee 0,\right. \\
& \left.\frac{1}{c}\left(x_{3}+y_{3}-1+(c-1) x_{3} y_{3}\right) \vee 0\right), c \in(0,1] ; a, b \geq 1 \text {, } \\
& S_{21}(x, y)=\left(\left(x_{1}^{a}+y_{1}^{a}-x_{1}^{a} y_{1}^{a}\right)^{\frac{1}{a}},\left(\left(x_{2}+y_{2}-1\right)(1+b)-b x_{2} y_{2}\right) \vee 0,\right. \\
& \left.\frac{1}{c}\left(x_{3}+y_{3}-1+(c-1) x_{3} y_{3}\right) \vee 0\right), a \geq 1 ; b \geq 0 ; c \in(0,1], \\
& S_{22}(x, y)=\left(\left(x_{1}^{a}+y_{1}^{a}-x_{1}^{a} y_{1}^{a}\right)^{\frac{1}{a}},\left(\left(x_{2}+y_{2}-1\right)(1+\lambda)-\lambda x_{2} y_{2}\right) \vee 0,\right. \\
& \left.0 \vee\left(x_{3}^{b}+y_{3}^{b}-1\right)^{\frac{1}{b}}\right), \lambda \geq 0, a, b \geq 1, \\
& S_{23}(x, y)=\left(\left(x_{1}^{a}+y_{1}^{a}-x_{1}^{a} y_{1}^{a}\right)^{\frac{1}{a}}, 0 \vee\left(x_{2}^{b}+y_{2}^{b}-1\right)^{\frac{1}{b}},\right. \\
& \left.\left(\left(x_{3}+y_{3}-1\right)(1+\lambda)-\lambda x_{3} y_{3}\right) \vee 0\right), \lambda \geq 0, a, b \geq 1 .
\end{aligned}
$$




\subsection{Strict-nipoltent-strict t-conorms subclass, denoted by $\nabla_{\text {sns }}$}

Definition 5.4. A picture fuzzy t-conorm $S$ is called strict-nipoltent-strict iff

$$
S(x, y)=\left(s_{3}\left(x_{1}, y_{1}\right), t_{2}\left(x_{2}, y_{2}\right), t_{1}\left(x_{3}, y_{3}\right)\right), \forall x, y \in D^{*},
$$

where $t_{1}$ is a strict fuzzy t-norm on $[0,1], t_{2}$ is a nipoltent fuzzy t-norm on $[0,1]$ and $s_{3}$ is a strict fuzzy t-conorm on $[0,1]$.

Examples 5.4.

$$
\begin{aligned}
& S_{24}(x, y)=\left(x_{1}+y_{1}-x_{1} y_{1}, 0 \vee\left(x_{2}+y_{2}-1\right), x_{3} y_{3}\right), \\
& S_{25}(x, y)=\left(\left(x_{1}^{a}+y_{1}^{a}-x_{1}^{a} y_{1}^{a}\right)^{\frac{1}{a}},\left(\left(x_{2}+y_{2}-1\right)\left(1+\lambda_{1}\right)-\lambda_{1} x_{2} y_{2}\right) \vee 0\right. \text {, } \\
& \left.\frac{x_{3} y_{3}}{\lambda_{2}+\left(1-\lambda_{2}\right)\left(x_{3}+y_{3}-x_{3} y_{3}\right)}\right), \lambda_{1} \geq 0 ; \lambda_{2}, a \geq 1, \\
& S_{26}(x, y)=\left(\left(x_{1}^{a}+y_{1}^{a}-x_{1}^{a} y_{1}^{a}\right)^{\frac{1}{a}}, 0 \vee\left(x_{2}^{b}+y_{2}^{b}-1\right)^{\frac{1}{b}}\right. \text {, } \\
& \left.\frac{x_{3} y_{3}}{\lambda_{1}+\left(1-\lambda_{1}\right)\left(x_{3}+y_{3}-x_{3} y_{3}\right)}\right), \lambda_{1} \geq 1 ; a, b \geq 1, \\
& S_{27}(x, y)=\left(\left(x_{1}^{a}+y_{1}^{a}-x_{x_{3}}^{a} y_{3}^{a}\right)^{\frac{1}{a}}, \frac{1}{b}\left(x_{2}+y_{2}-1+(b-1) x_{2} y_{2}\right) \vee 0,\right. \\
& \left.\frac{x_{3} y_{3}}{\lambda_{1}+\left(1-\lambda_{1}\right)\left(x_{3}+y_{3}-x_{3} y_{3}\right)}\right), a, \lambda_{1} \geq 1 ; b \in(0,1] \text {. }
\end{aligned}
$$

\subsection{Strict-strict-nipoltent t-conorms subclass, denoted by $\nabla_{s s n}$}

Definition 5.5. A picture fuzzy t-conorm $S$ is called strict-strict-nipoltent iff

$$
S(x, y)=\left(s_{3}\left(x_{1}, y_{1}\right), t_{2}\left(x_{2}, y_{2}\right), t_{1}\left(x_{3}, y_{3}\right)\right), \forall x, y \in D^{*},
$$

where $t_{1}$ is a nipoltent fuzzy t-norm on $[0,1], t_{2}$ is a strict fuzzy t-norm on $[0,1]$ and $s_{3}$ is a strict fuzzy t-conorm on $[0,1]$.

\section{Examples 5.5.}

$$
\begin{gathered}
S_{28}(x, y)=\left(x_{1}+y_{1}-x_{1} y_{1}, x_{2} y_{2}, 0 \vee\left(x_{3}+y_{3}-1\right)\right), \\
S_{29}(x, y)=\left(\left(x_{1}^{a}+y_{1}^{a}-x_{1}^{a} y_{1}^{a}\right)^{\frac{1}{a}}, \frac{x_{2} y_{2}}{\lambda+(1-\lambda)\left(x_{2}+y_{2}-x_{2} y_{2}\right)},\right. \\
\left.\frac{1}{b}\left(x_{3}+y_{3}-1+(b-1) x_{3} y_{3}\right) \vee 0\right), a, \lambda \geq 1 ; b \in(0,1], \\
S_{30}(x, y)=\left(\left(x_{1}^{a}+y_{1}^{a}-x_{1}^{a} y_{1}^{a}\right)^{\frac{1}{a}}, \frac{x_{2} y_{2}}{\lambda+(1-\lambda)\left(x_{2}+y_{2}-x_{2} y_{2}\right)},\right. \\
\left.0 \vee\left(x_{3}^{b}+y_{3}^{b}-1\right)^{\frac{1}{b}}\right), a, b, \lambda \geq 1, \\
S_{31}(x, y)=\left(\left(x_{1}^{a}+y_{1}^{a}-x_{1}^{a} y_{1}^{a}\right)^{\frac{1}{a}}, \frac{x_{2} y_{2}}{\lambda+(1-\lambda)\left(x_{2}+y_{2}-x_{2} y_{2}\right)},\right. \\
\left.\left(\left(x_{2}+y_{2}-1\right)(1+b)-b x_{2} y_{2}\right) \vee 0\right), a, \lambda \geq 1 ; b \geq 0 .
\end{gathered}
$$

Proposition 5.6. There doesn't exist representable picture fuzzy t-conorm $S$

$$
S(x, y)=\left(s_{3}\left(x_{1}, y_{1}\right), t_{2}\left(x_{2}, y_{2}\right), t_{1}\left(x_{3}, y_{3}\right)\right), \forall x, y \in D^{*},
$$


here $t_{1}$ or $t_{2}$ is strict fuzzy t-norm on $[0,1]$ and $s_{3}$ is a nipoltent fuzzy $t$-conorm on $[0,1]$.

Proposition 5.7. If $S$ belongs to one of four classes $\nabla_{\text {sss }}, \nabla_{\text {snn }}, \nabla_{\text {sns }}, \nabla_{\text {ssn }}$ then $S$ is strict.

Proposition 5.8. If $S$ belongs to the class $\nabla_{n n n}$ then $S$ is nipoltent.

\section{SOME NEW DE MORGAN PICTURE OPERATOR TRIPLES IN PICTURE FUZZY LOGIC}

De Morgan picture operator triples is a basic algebra of the Picture Fuzzy Logic. The notion of t-norm plays the role of intersection, or in logical terms, "and". The duality of that notion is that of union, or "or" . In the case of sets, union and intersection are related via complements. The well-known De Morgan formulas do that. They are

$$
(A \cup B)^{C}=\left(A^{C} \cap B^{C}\right),(A \cap B)^{C}=\left(A^{C} \cup B^{C}\right) .
$$

Let $T(x, y)$ be a picture fuzzy t-norm and let $S(x, y)$ be a picture fuzzy t-conorm and $n(x)$ be a picture negation operator, The De Morgan formulas now become the new equations

$$
\begin{aligned}
& n(S(x, y))=T(n(x), n(y)), \forall x, y \in D^{*}, \\
& n(T(x, y))=S(n(x), n(y)), \forall x, y \in D^{*} .
\end{aligned}
$$

Definition 6.1. The triple of operators $(T, S, n)$ is called a De Morgan picture operator triple if they satisfy both the equation $(a, *)$ and the equation $(b, *)$. Then we say that $T$ and $S$ are dual corresponding to the negation operator $n(x)$.

With an involutive picture negation operator, De Morgan triples of picture fuzzy operators satisfy the following equations

$$
S(x, y)=n(T(n(x), n(y))), \forall x, y \in D^{*},
$$

and

$$
T(x, y)=n(S(n(x), n(y))), \forall x, y \in D^{*} .
$$

Some De Morgan picture operator triples were given in [9].

Now we give some new De Morgan picture operator triples $\left(T, S, n_{0}\right)$ corresponding the picture negation operator $n_{0}(x)$.

Proposition 6.2. The triple $\left(T_{\min }, S_{\max }, n_{0}\right)$ corresponding the picture negation operator $n_{0}(x)$ is a De Morgan picture operator triple.

Proof. We have

$$
T_{\min }(x, y)=\left(\min \left(x_{1}, y_{1}\right), \min \left(x_{2}, y_{2}\right), \max \left(x_{3}, y_{3}\right)\right)
$$

And

$$
\begin{aligned}
S_{\max }(x, y) & =\left(\max \left(x_{1}, y_{1}\right), \min \left(x_{2}, y_{2}\right), \min \left(x_{3}, y_{3}\right)\right) . \\
n_{0}\left(S_{\max }(x, y)\right) & =n_{0}\left(\max \left(x_{1}, y_{1}\right), \min \left(x_{2}, y_{2}\right), \min \left(x_{3}, y_{3}\right)\right) \\
& =\left(\min \left(x_{3}, y_{3}\right), 0, \max \left(x_{1}, y_{1}\right)\right),
\end{aligned}
$$

and

$$
\begin{aligned}
& n_{0}(x)=\left(x_{3}, 0, x_{1}\right), n_{0}(y)=\left(y_{3}, 0, y_{1}\right) \Rightarrow \\
& \begin{aligned}
T_{\min }\left(n_{0}(x), n_{0}(y)\right) & =\left(\min \left(x_{3}, y_{3}\right), \min (0,0), \max \left(x_{1}, y_{1}\right)\right) \\
& =\left(\min \left(x_{3}, y_{3}\right), 0, \max \left(x_{1}, y_{1}\right)\right)=n_{0}\left(S_{\max }(x, y)\right) .
\end{aligned}
\end{aligned}
$$


It means that we have the equation $(a, *)$. Analogously

$$
\begin{gathered}
n_{0}\left(T_{\min }(x, y)\right)=\left(\max \left(x_{3}, y_{3}\right), 0, \min \left(x_{1}, y_{1}\right)\right), \\
S_{\max }\left(n_{0}(x), n_{0}(y)\right)=\left(\max \left(x_{3}, y_{3}\right), \min (0,0), \operatorname{mn}\left(x_{1}, y_{1}\right)\right) \\
=\left(\max \left(x_{3}, y_{3}\right), 0, \min \left(x_{1}, y_{1}\right)\right)=n_{0}\left(T_{\min }(x, y)\right) .
\end{gathered}
$$

We have the equation $(b, *)$.

Proposition 6.3. Consider the picture t-norm $T_{02}(x, y)=\left(\min \left(x_{1}, y_{1}\right), x_{2} y_{2}, \max \left(x_{3}, y_{3}\right)\right)$ and the picture $t$-conorm $S_{02}(x, y)=\left(\max \left(x_{1}, y_{1}\right), x_{2} y_{2}, \min \left(x_{3}, y_{3}\right)\right)$.

The triple $\left(T_{02}, S_{02}, n_{0}\right)$ is a De Morgan picture operator triple.

Proof. The proof is analogous to the proof of the Proposition 6.2.

Proposition 6.4. Let $t_{2}(x, y), t_{3}(x, y)$ be fuzzy t-norms on $[0,1]$. Consider the picture fuzzy t-norm

$$
T_{\min , t_{2}}(x, y)=\left(\min \left(x_{1}, y_{1}\right), t_{2}\left(x_{2} y_{2}\right), \max \left(x_{3}, y_{3}\right)\right)
$$

and the picture fuzzy $t$-conrm

$$
S_{\max , t_{3}}(x, y)=\left(\max \left(x_{1}, y_{1}\right), t_{2}\left(x_{2} y_{2}\right), \min \left(x_{3}, y_{3}\right)\right) .
$$

The triple of operators $\left(T_{\min , t_{2}}, S_{\max , t_{3}}, n_{0}\right)$ corresponding the picture negation operator $n_{0}(x)$ is a De Morgan picture operator triple.

Proof.

$$
\begin{aligned}
S_{\max , t_{3}}(x, y)=\left(\max \left(x_{1}, y_{1}\right), t_{3}\left(x_{2}, y_{2}\right), \min \left(x_{3}, y_{3}\right)\right) . \\
\begin{aligned}
n_{0}\left(S_{\text {max }, t_{3}}(x, y)\right) & =n_{0}\left(\max \left(x_{1}, y_{1}\right), t_{3}\left(x_{2}, y_{2}\right), \min \left(x_{3}, y_{3}\right)\right) \\
& =\left(\min \left(x_{3}, y_{3}\right), 0, \max \left(x_{1}, y_{1}\right)\right),
\end{aligned}
\end{aligned}
$$

and

$$
\begin{aligned}
& n_{0}(x)=\left(x_{3}, 0, x_{1}\right), n_{0}(y)=\left(y_{3}, 0, y_{1}\right) \Rightarrow \\
& \begin{aligned}
T_{\min , t_{2}}\left(n_{0}(x), n_{0}(y)\right) & =\left(\min \left(x_{3}, y_{3}\right), t_{2}(0,0), \max \left(x_{1}, y_{1}\right)\right) \\
& =\left(\min \left(x_{3}, y_{3}\right), 0, \max \left(x_{1}, y_{1}\right)\right)=n_{0}\left(S_{\max , t_{3}}(x, y)\right) .
\end{aligned}
\end{aligned}
$$

It means that we have the equation $(a, *)$. Analogously

$$
\begin{aligned}
n_{0}\left(T_{\min , t_{2}}(x, y)\right)=\left(\max \left(x_{3}, y_{3}\right), 0, \min \left(x_{1}, y_{1}\right)\right), \\
S_{\text {max }, t_{3}}\left(n_{0}(x), n_{0}(y)\right)=\left(\max \left(x_{3}, y_{3}\right), t_{3}(0,0), \operatorname{mn}\left(x_{1}, y_{1}\right)\right) \\
=\left(\max \left(x_{3}, y_{3}\right), 0, \min \left(x_{1}, y_{1}\right)\right)=n_{0}\left(T_{\min , t}(x, y)\right) .
\end{aligned}
$$

We have the equation $(b, *)$.

We easily receive the following proposition.

Proposition 6.5. Consider the picture t-norm $T_{04}$ and the picture $t$-conorm $S_{04}$

and

$$
T_{04}(x, y)=\left(x_{1} y_{1}, x_{2} y_{2}, x_{3}+y_{3}-x_{3} y_{3}\right),
$$

$$
S_{04}(x, y)=\left(x_{1}+y_{1}-x_{1} y_{1}, x_{2} y_{2}, x_{3} y_{3}\right) .
$$

The triple $\left(T_{04}, S_{04}, n_{0}\right)$ is a De Morgan picture operator triple. 
Proof.

and

$$
\begin{aligned}
n_{0}\left(S_{04}(x, y)\right) & =n_{0}\left(x_{1}+y_{1}-x_{1} y_{1}, x_{2} y_{2}, x_{3} y_{3}\right) \\
& =\left(x_{3} y_{3}, 0, x_{1}+y_{1}-x_{1} y_{1}\right),
\end{aligned}
$$

$n_{0}(x)=\left(x_{3}, 0, x_{1}\right), n_{0}(y)=\left(y_{3}, 0, y_{1}\right) \Rightarrow$

$T_{04}\left(n_{0}(x), n_{0}(y)\right)=\left(x_{3} y_{3}, 0.0, x_{1}+y_{1}-x_{1} y_{1}\right)=\left(x_{3} y_{3}, 0, x_{1}+y_{1}-x_{1} y_{1}\right)=n_{0}\left(S_{04}(x, y)\right)$.

It means that we have the equation $(a, *)$. Analogously

$$
\begin{gathered}
n_{0}\left(T_{04}(x, y)\right)=\left(x_{3}+y_{3}-x_{3} y_{3}, 0, x_{1} y_{1}\right), \\
S_{04}\left(n_{0}(x), n_{0}(y)\right)=\left(x_{3}+y_{3}-x_{3} y_{3}, 0.0, x_{1} y_{1}\right) \\
=\left(x_{3}+y_{3}-x_{3} y_{3}, 0, x_{1} y_{1}\right)=n_{0}\left(T_{04}(x, y)\right) .
\end{gathered}
$$

We have the equation $(b, *)$.

Now we consider the case where picture t-norm $T$ belongs to the nilpotent, nilpotent, nilpotent subclass $\Delta_{n n n}$ and $S$ belongs to the subclass $\nabla_{n n n}$.

Proposition 6.6. Consider the picture t-norm $T_{3}$ and the picture $t$-conorm $S_{3}$

$$
\begin{aligned}
& T_{3}(x, y)=\left(0 \vee\left(x_{1}+y_{1}-1\right), 0 \vee\left(x_{2}+y_{2}-1\right), 1 \wedge\left(x_{3}+y_{3}\right)\right), \\
& S_{3}(x, y)=\left(1 \wedge\left(x_{1}+y_{1}\right), 0 \vee\left(x_{2}+y_{2}-1\right), 0 \vee\left(x_{3}+y_{3}-1\right)\right) .
\end{aligned}
$$

The triple $\left(T_{3}, S_{3}, n_{0}\right)$ is a De Morgan picture operator triple.

Proof.

$$
\begin{aligned}
& \begin{aligned}
n_{0}\left(S_{3}(x, y)\right)= & n_{0}\left(\left(1 \wedge\left(x_{1}+y_{1}\right), 0 \vee\left(x_{2}+y_{2}-1\right), 0 \vee\left(x_{3}+y_{3}-1\right)\right)\right) \\
= & \left(0 \vee\left(x_{3}+y_{3}-1\right), 0,\left(1 \wedge\left(x_{1}+y_{1}\right)\right),\right.
\end{aligned} \\
& \begin{aligned}
n_{0}(x)=\left(x_{3}, 0, x_{1}\right), & n_{0}(y)=\left(y_{3}, 0, y_{1}\right) \Rightarrow \\
T_{3}\left(n_{0}(x), n_{0}(y)\right)= & \left.\left(\left(\left(x_{3}+y_{3}-1\right) \vee 0\right),(0+0-1) \vee 0\right), 1 \wedge\left(x_{1}+y_{1}\right)\right) \\
= & \left(\left(0 \vee\left(x_{3}+y_{3}-1\right), 0,1 \wedge\left(x_{1}+y_{1}\right)\right)=n_{0}\left(S_{3}(x, y)\right) .\right.
\end{aligned}
\end{aligned}
$$

It means that we have the equation $(a, *)$. Analogously

$$
\begin{aligned}
n_{0}\left(T_{3}(x, y)\right) & =\left(1 \wedge\left(x_{3}+y_{3}\right)\right), 0,\left(0 \vee\left(x_{1}+y_{1}-1\right)\right), \\
S_{3}\left(n_{0}(x), n_{0}(y)\right)= & \left(1 \wedge\left(x_{3}+y_{3}\right),\left(0 \vee(0+0-1),\left(0 \vee\left(x_{1}+y_{1}-1\right)\right)\right.\right. \\
= & \left(1 \wedge\left(x_{3}+y_{3}\right), 0,\left(0 \vee\left(x_{1}+y_{1}-1\right)\right)=n_{0}\left(T_{3}(x, y)\right) .\right.
\end{aligned}
$$

We have the equation $(b, *)$.

Proposition 6.7. Consider the picture t-norm $T_{4}$ of subclass $\Delta_{n n n}$ and $S_{4}$ belongs to the subclass $\nabla_{n n n}$.

$T_{4}(x, y)=\left(\left(\left(x_{1}+y_{1}-1\right)\left(1+\lambda_{1}\right)-\lambda_{1} x_{1} y_{1}\right) \vee 0,\left(\left(x_{2}+y_{2}-1\right)\left(1+\lambda_{2}\right)-\lambda_{2} x_{2} y_{2}\right) \vee 0,1 \wedge\right.$ $\left.\left(x_{3}^{a}+y_{3}^{a}\right)^{\frac{1}{a}}\right), S_{4}(x, y)=\left(1 \wedge\left(x_{1}^{a}+y_{1}^{a}\right)^{\frac{1}{a}},\left(\left(x_{2}+y_{2}-1\right)\left(1+\lambda_{2}\right)-\lambda_{2} x_{2} y_{2}\right) \vee 0,\left(\left(x_{3}+y_{3}-1\right)(1+\right.\right.$ $\left.\left.\left.\lambda_{1}\right)-\lambda_{1} x_{3} y_{3}\right) \vee 0\right)$, where $\lambda_{1}, \lambda_{2} \in[0,+\infty), a \geq 1$. The triple $\left(T_{4}, S_{4}, n_{0}\right)$ is a De Morgan picture operator triple. 
Proof.

$$
\begin{aligned}
& n_{0}\left(S_{4}(x, y)\right) \\
& =n_{0}\left(\left(1 \wedge\left(x_{1}^{a}+y_{1}^{a}\right)^{\frac{1}{a}},\left(\left(x_{2}+y_{2}-1\right)\left(1+\lambda_{2}\right)-\lambda_{2} x_{2} y_{2}\right) \vee 0,\left(\left(x_{3}+y_{3}-1\right)\left(1+\lambda_{1}\right)-\lambda_{1} x_{3} y_{3}\right) \vee 0\right)\right) \\
& \left.=\left(\left(x_{3}+y_{3}-1\right)\left(1+\lambda_{1}\right)-\lambda_{1} x_{3} y_{3}\right) \vee 0\right), 0,\left(1 \wedge\left(x_{1}^{a}+y_{1}^{a}\right)^{\frac{1}{a}}\right) \\
& T_{4}\left(n_{0}(x), n_{0}(y)\right) \\
& =\left(\left(\left(x_{3}+y_{3}-1\right)\left(1+\lambda_{1}\right)-\lambda_{1} x_{3} y_{3}\right) \vee 0,\left((0+0-1)\left(1+\lambda_{2}\right)-\lambda_{2} 0.0\right) \vee 0,1 \wedge\left(x_{1}^{a}+y_{1}^{a}\right)^{\frac{1}{a}}\right) \\
& =\left(\left(\left(x_{3}+y_{3}-1\right)\left(1+\lambda_{1}\right)-\lambda_{1} x_{3} y_{3}\right) \vee 0,0,1 \wedge\left(x_{1}^{a}+y_{1}^{a}\right)^{\frac{1}{a}}\right)=n_{0}\left(S_{4}(x, y)\right) .
\end{aligned}
$$

It means that we have the equation $(a, *)$. Analogously

$$
\left.\left.n_{0}\left(T_{4}(x, y)\right)=\left(\left(1 \wedge\left(x_{3}^{a}+y_{3}^{a}\right)^{\frac{1}{a}}\right), 0,\left(x_{1}+y_{1}-1\right)\left(1+\lambda_{1}\right)-\lambda_{1} x_{1} y_{1}\right) \vee 0\right)\right),
$$

$S_{4}\left(n_{0}(x), n_{0}(y)\right)$

$$
\begin{aligned}
& \left.=\left(\left(1 \wedge\left(x_{3}^{a}+y_{3}^{a}\right)^{\frac{1}{a}},\left((0+0-1)\left(1+\lambda_{2}\right)-\lambda_{2} 0.0\right)\right) \vee 0,\left(\left(x_{1}+y_{1}-1\right)\left(1+\lambda_{1}\right)-\lambda_{1} x_{1} y_{13}\right) \vee 0\right)\right) \\
& =\left(\left(1 \wedge\left(x_{3}^{a}+y_{3}^{a}\right)^{\frac{1}{a}}, 0,\left(\left(x_{1}+y_{1}-1\right)\left(1+\lambda_{1}\right)-\lambda_{1} x_{1} y_{13}\right) \vee 0\right)\right)=n_{0}\left(T_{4}(x, y)\right) .
\end{aligned}
$$

We have the equation $(b, *)$.

Proposition 6.8. We consider the case picture t-norm $T_{11}$ belongs to $\Delta_{n n s}$ - the nilpotent, nilpotent, strict subclass and $S_{11}$ belongs to the subclass $\nabla_{\text {snn }}$.

$$
\begin{aligned}
& T_{11}(x, y)=\left(0 \vee\left(x_{1}+y_{1}-1\right), 0 \vee\left(x_{2}+y_{2}-1\right), x_{3}+y_{3}-x_{3} y_{3}\right), \\
& S_{11}(x, y)=\left(x_{1}+y_{1}-x_{1} y_{1}, 0 \vee\left(x_{2}+y_{2}-1\right), 0 \vee\left(x_{3}+y_{3}-1\right)\right) .
\end{aligned}
$$

The triple $\left(T_{11}, S_{11}, n_{0}\right)$ is a De Morgan picture operator triple.

Proof.

$$
\begin{aligned}
n_{0}\left(S_{11}(x, y)\right)= & n\left(\left(x_{1}+y_{1}-x_{1} y_{1}, 0 \vee\left(x_{2}+y_{2}-1\right), 0 \vee\left(x_{3}+y_{3}-1\right)\right)\right) \\
= & \left(\left(0 \vee\left(x_{3}+y_{3}-1\right)\right), 0,\left(x_{1}+y_{1}-x_{1} y_{1}\right)\right), \\
T_{11}\left(n_{0}(x), n_{0}(y)\right) & =\left(0 \vee\left(x_{3}+y_{3}-1\right), 0 \vee(0+0-1), x_{1}+y_{1}-x_{1} y_{1}\right) \\
& =\left(0 \vee\left(x_{3}+y_{3}-1\right), 0, x_{1}+y_{1}-x_{1} y_{1}\right) \\
& =n_{0}\left(S_{11}(x, y)\right) .
\end{aligned}
$$

It means that we have the equation $(a, *)$. Analogously

$$
\begin{aligned}
n_{0}\left(T_{11}(x, y)\right)= & n_{0}\left(\left(0 \vee\left(x_{1}+y_{1}-1\right), 0 \vee\left(x_{2}+y_{2}-1\right), x_{3}+y_{3}-x_{3} y_{3}\right)\right) \\
= & \left(\left(x_{3}+y_{3}-x_{3} y_{3}\right), 0,\left(0 \vee\left(x_{1}+y_{1}-1\right)\right),\right. \\
S_{11}\left(n_{0}(x), n_{0}(y)\right) & =\left(\left(x_{3}+y_{3}-x_{3} y_{3}\right), 0 \vee(0+0-1), 0 \vee\left(x_{1}+y_{1}-1\right)\right) \\
& =\left(\left(x_{3}+y_{3}-x_{3} y_{3}\right), 0,\left(0 \vee\left(x_{1}+y_{1}-1\right)\right)=n_{0}\left(T_{11}(x, y)\right),\right.
\end{aligned}
$$

we have the equation $(b, *)$.

Now we consider the case where picture t-norm $T_{12}$ belongs the nilpotent, nilpotent, strict subclass $\Delta_{n n s}$ and the picture t-conorm $S_{12}$ belongs to the subclass $\nabla_{s n n}$. 
Proposition 6.9. Consider

$$
\begin{aligned}
& T_{12}(x, y)=\left(\frac{1}{2}\left(x_{1}+y_{1}-1+x_{1} y_{1}\right) \vee 0, \frac{1}{2}\left(x_{2}+y_{2}-1+x_{2} y_{2}\right) \vee 0,\left(x_{3}^{a}+y_{3}^{a}-x_{3}^{a} y_{3}^{a}\right)^{\frac{1}{a}}\right), \\
& S_{12}(x, y)=\left(\left(x_{1}^{a}+y_{1}^{a}-x_{1}^{a} y_{1}^{a}\right)^{\frac{1}{a}}, \frac{1}{2}\left(x_{2}+y_{2}-1+x_{2} y_{2}\right) \vee 0, \frac{1}{2}\left(x_{3}+y_{3}-1+x_{3} y_{3}\right) \vee 0\right),
\end{aligned}
$$

where $a \geq 1$.

The triple $\left(T_{12}, S_{12}, n_{0}\right)$ is a De Morgan picture operator triple.

Proof.

$$
\begin{aligned}
n_{0} & \left(S_{12}(x, y)\right) \\
= & n_{0}\left(\left(\left(x_{1}^{a}+y_{1}^{a}-x_{1}^{a} y_{1}^{a}\right)^{\frac{1}{a}}, \frac{1}{2}\left(x_{2}+y_{2}-1+x_{2} y_{2}\right) \vee 0, \frac{1}{2}\left(x_{3}+y_{3}-1+x_{3} y_{3}\right) \vee 0\right)\right) \\
= & \left(\left(\frac{1}{2}\left(x_{3}+y_{3}-1+x_{3} y_{3}\right) \vee 0\right), 0,\left(x_{1}^{a}+y_{1}^{a}-x_{1}^{a} y_{1}^{a}\right)^{\frac{1}{a}}\right), \\
& T_{12}\left(n_{0}(x), n_{0}(y)\right) \\
= & \left.\left(\left(\frac{1}{2}\left(x_{3}+y_{31}-1+x_{3} y_{3}\right) \vee 0\right), \frac{1}{2}(0+0-1+0.0) \vee 0,\left(x_{1}^{a}+y_{1}^{a}-x_{1}^{a} y_{1}^{a}\right)^{\frac{1}{a}}\right)\right) \\
& =\left(\left(\frac{1}{2}\left(x_{3}+y_{31}-1+x_{3} y_{3}\right) \vee 0\right), 0,\left(x_{1}^{a}+y_{1}^{a}-x_{1}^{a} y_{1}^{a}\right)^{\frac{1}{a}}\right)=n_{0}\left(S_{12}(x, y)\right) .
\end{aligned}
$$

It means that we have the equation $(a, *)$. Analogously

$$
\begin{aligned}
& n_{0}\left(T_{12}(x, y)\right) \\
& \left.=n_{0}\left(\left(\frac{1}{2}\left(x_{1}+y_{1}-1+x_{1} y_{1}\right) \vee 0\right), \frac{1}{2}\left(x_{2}+y_{2}-1+x_{2} y_{2}\right) \vee 0,\left(x_{3}^{a}+y_{3}^{a}-x_{3}^{a} y_{3}^{a}\right)^{\frac{1}{a}}\right)\right) \\
& \left.=\left(\left(x_{3}^{a}+y_{3}^{a}-x_{3}^{a} y_{3}^{a}\right)^{\frac{1}{a}}\right), 0,\left(\frac{1}{2}\left(x_{1}+y_{1}-1+x_{1} y_{1}\right) \vee 0\right)\right)=S_{12}\left(n_{0}(x), n_{0}(y)\right),
\end{aligned}
$$

$S_{12}\left(n_{0}(x), n_{0}(y)\right)$

$=\left(\left(x_{3}^{a}+y_{3}^{a}-x_{3}^{a} y_{3}^{a}\right)^{\frac{1}{a}},\left(\frac{1}{2}(0+0-1+0.0) \vee 0\right),\left(\frac{1}{2}\left(x_{1}+y_{1}-1+x_{1} y_{3}\right) \vee 0\right)\right)$

$=\left(\left(x_{3}^{a}+y_{3}^{a}-x_{3}^{a} y_{3}^{a}\right)^{\frac{1}{a}}, 0,\left(\frac{1}{2}\left(x_{1}+y_{1}-1+x_{1} y_{3}\right) \vee 0\right)\right)=n_{0}\left(T_{12}(x, y)\right)$.

It means that we have the equation $(b, *)$.

Some other De Morgan picture operator triples can be seen in $[9,8]$.

\section{CONCLUSION}

Conjunction operations (fuzzy t-norms) and disjunction operations (fuzzy t-conorms) are basic operators of the fuzzy logics $[22,13]$. Picture fuzzy t-norms and picture fuzzy t-conorms firstly were defined and studied in 2015 [6,9]. In this paper we give some algebraic properties of the picture fuzzy t-norms and the picture fuzzy t-conrms on picture fuzzy sets, including some classes of representable picture fuzzy t-norms and and some classes of representable picture fuzzy t-conorms. Then we study the De Morgan picture operator triples of the Picture Fuzzy Logics. Some new classes of De Morgan picture operator triples were presented. In the following papers new other issues of the Picture Fuzzy Logic should be considered. 


\section{Acknowledgment}

This research is funded by the Vietnam National Foundation for Science and Technology Development (NAFOSTED) under grant number 102.01-2017.02.

\section{REFERENCES}

[1] K. Atanassov, "Intuitionistic fuzzy sets," Fuzzy Sets and Systems, vol. 20, pp. 87-96, 1986.

[2] — On Intuitionistic Fuzzy Sets Theory. Berlin: Springer, 2012.

[3] B. C. Cuong, "Picture fuzzy sets - first results," in Part 1, Seminar: Neuro-Fuzzy Systems with Applications, Preprint 03/2013, May 2013, Institute of Mathematics, Hanoi, Vietnam, 2013.

[4] —_ "Picture fuzzy sets - first results," in Part 2, Seminar: Neuro-Fuzzy Systems with Applications, Preprint 04/2013, June 2013, Institute of Mathematics, Hanoi Vietnam, 2013.

[5] —_, "Picture fuzzy sets," Journal of Computer Science and Cybernetics, vol. 30, no. 4, pp. 409-420, 2014.

[6] B. C. Cuong and P. V. Hai, "Some fuzzy logic operators for picture fuzzy sets," in the Proceedings of the 2015 IEEE International Conference on Knowledge Systems Engineering, KSE 2015, IEEE Computer Society Publications and CPS, Washington, 2015, pp. 132-137.

[7] B. C. Cuong and V. Kreinovich, "Picture fuzzy sets - a new concept for computational intelligence problems," in The Proceedings of the World Congress on Information and Communication Technologies (WICT 2013), Hanoi, Vietnam, IEEE CS, 2013, pp. 1-6.

[8] B. C. Cuong and R. T. Ngan, "Some new de morgan triples of operators in the picture fuzzy sets theory," in Seminar: Neuro-Fuzzy Systems with Applications, Preprint 08/2017, June 2017, Institute of Mathematics, Hanoi, Vietnam, 2017.

[9] B. C. Cuong, R. T. Ngan, and B. D. Hai, "An involutive picture fuzzy negation on picture fuzzy sets and some de morgan triples," in the Proceedings of the 2015 IEEE International Conference on Knowledge and Systems Engineering, KSE 2015, IEEE Computer Society Publications and CPS, Washington, 2015, pp. 126-131.

[10] G. Deschrijver, C. Cornelis, and E. E. Kerre, "On the representation of intuitionistic fuzzy t-norms and t-conorms," IEEE Transactions on Fuzzy Systems, vol. 12, no. 1, pp. 45-61, 2004.

[11] F.Smaranddache, A unifying field in logics. Neutrosophy: Neutrosophic probability, set and logic. Rehoboth: American Research Press, 1999.

[12] J. Goguen, "L-fuzzy sets," J. Math. Anal. Appl., vol. 18, pp. 145-174, 1967.

[13] E. P. Klement and R. Mesiar, Logical, Algebraic, Analytic and Probabilistic Aspects of Triangular Norms. Amsterdam: Elsevie, 2005.

[14] P. H. Phong and B. C. Cuong, "Some intuitionistic linguistic aggregation operators," Journal of Computer Science and Cybernetics, vol. 30, no. 3, pp. 216-226, 2014.

[15] —_, "Multi-criteria group decision making with picture linguistic numbers," VNU Journal of Science: Computer Science and Communication Engineering, vol. 32, no. 3, pp. 38-51, 2016.

[16] _ _ "Symbolic computational models for intuitionistic linguistic information," Journal of Computer Science an Cybernetics, vol. 32, no. 1, pp. 30-44, 2016. 
[17] L. H. Son, "DPFCM: A novel distributed picture fuzzy clustering method on picture fuzzy sets," Expert systems with applications, vol. 42, pp. 51-66, 2015.

[18] —_ "Generalized picture distance measure and applications to picture fuzzy clustering," $A p$ plied Soft Computing, vol. 46, p. 284295, 2016.

[19] _ - "Measuring analogousness in picture fuzzy sets: From picture distance measures to picture association, measures," Fuzzy Optimization and Decision Making, pp. 1-20, 2017.

[20] L. H. Son and P. H. Thong, "Some novel hybrid forecast methods based on picture fuzzy clustering for weather nowcasting from satellite image sequences," Applied Intelligence, vol. 46, no. 1, pp. 1-15, 2017.

[21] L. H. Son, P. V. Viet, and P. V. Hai, "Picture inference system: A new fuzzy inference system on picture fuzzy set," Applied Intelligence, vol. 46, no. 3, pp. 652-669, 2017.

[22] T.H.Nguyen and E.Walker, A first course in fuzzy logic, Second Edition. CRC, 2000, Boca Raton: Chapman \& Hall, 2000.

[23] P. H. Thong and L. H. Son, "Picture fuzzy clustering: A new computational intelligence method," Soft Computing, vol. 20, no. 9, pp. 3544-3562, 2016.

[24] C. Wang, X. Zhou, H. Tu, and S. Tao, "Some geometric aggregation operators based on picture fuzzy sets and their application in multiple attribute decision making," Italian Journal of Pure and Applied Mathematics, no. 37, pp. 477-492, 2017.

[25] G. Wei, "Picture fuzzy cross-entropy for multiple attribute decision making problems," Journal of Business Economics and Management, vol. 17, no. 4, pp. 491-502, 2016.

[26] — , "Picture 2-tuple linguistic bonferroni mean operators and their application to multiple attribute decision making," International Journal of Fuzzy Systems, vol. 19, no. 4, pp. 9971010, 2017.

[27] L. A. Zadeh, "Fuzzy sets," Information and Control, vol. 8, pp. 338-353, 1965.

[28] — - "The concept of a linguistic variable and its application to approximate reasoning," Information Sciences, vol. 8, pp. 199-249, 1975.

Received on September 19, 2017

Revised on December 01, 2017 